\title{
The Brunswik Soeiety
}

\section{Newsletter}

Vol. 28, Nov, 2013

Many thanks to all authors for their contributions

To get information about future Newsletters you are welcome to join (free of charge) the email list of the Brunswik Society at: 


\section{Table of Contents}

\section{Foreword}

Kaufmann, E., Athanasou, J. A., \& Hamm, R. M.

\section{Contributions}

Afonso, J., \& Mesquita, I.

Designing a Protocol for Monitoring Tactical Decision-Making in Volleyball while Using a

Representative Design

Athanasou, J. A.

Interests as a Component of Adult Course Preferences.

Beckstead, J. W.

News from the Past Year.

Benbenishty, $R$.

A Personal Journey in the Land of Brunswik and Colleagues

Brückner, S.

Construct-irrelevant Mental Processes in University Students' Responding to Business and Economic Test Items: Using Symmetry Based on Verbal Reports to Establish the Validity of Test Score Interpretations.

Dhami, M. K.

The Cognitive Continuum and More on Bounded Rationality.....

Feigh, $K$.

Summary of Toward a Characterization of Adaptive Systems: A Framework for

Researchers and System Designers

Harries, $P$.

Researching Expert Decision Making to Develop Online Training for Novices

Huttin, $C$.

A Contribution to the Debate on Coherence versus Correspondence: Examples of Incoherencies in Regulated Medical Markets.

Kaplan, R. M.

Mobile Technologies and the Promise of Representative Design and Representative

Sampling

Kaufmann, E.

Interest in Brunswikian Literature?

Kaufmann, E., Reips, U.-D., \& Wittmann, W. W.

A Critical Meta-Analysis of Lens Model Studies in Human Judgment and Decision-Making. 
Mumpower, J. L.

News from the Past Year

Rolison, J. J.

Cue Polarity Effects in Learning: A "Matching Bias" for Multiple Cue Judgment.

Schweizer, G., Plessner, H., \& Brand, R.

Improving Referees' Decisions in Sports

Seifert, M., \& Hadida, A. L.

On the Relative Importance of Linear Model and Human Judge(s) in Combined

Forecasting...

Sjödahl, L.

Path dependency and Routines: A Threat to Capability Development

Stopfer, J. M., Egloff, B., Nestler, S., \& Back, M. D.

Using Lens Model Analyses to Understand Impression Formation in Online Social

Networks...

Unkelbach, C., \& Greifeneder, R.

A Lens Model Approach to Explain Fluency Effects in Judgments and Decisions 


\section{The Brunswik Society News/etter, 2013}

\section{Foreword}

After six years with Lars Sjödahl as editor of the Brunswik Society Newsletter, we have now taken over this task.

At the outset, as we assumed this responsibility we realized that it needed more than one person to do the job as well. So, we commenced as a group of three and have tried our best to come close to previous Newsletters. However, the foundation of this Newsletter is not the editorial team, it is the authors. Hence, we greatly appreciate and thank them for their contribution to the Newsletter this year.

As you can see, in the end we received varied contributions, from doctoral students and junior researchers as well as from senior researchers and emeritus professors. So, we have covered the entire academic spectrum with the content of these contributions.

Within this newsletter you will realize that Brunswikian research is a topic in sport psychology and also goes into the future with online social networks. Some of the contributions inform us about on-going research, others about published papers and one contribution goes even into the past, informing us about Brunswikian research in the early days.

We hope that the richness of the included contributions of this year's Newsletter inspires further thoughts for research while reading it.

Sincerely,

Esther Kaufmann, James A. Athanasou and Robert M. Hamm

Thank you to the webmaster of the Brunswik Society Tom Stewart for providing web access to the Newsletter. 


\title{
The Brunswik Society News/etter, 2013
}

\section{Contributions}

\section{Designing a Protocol for Monitoring Tactical Decision-Making in Volleyball while Using a Representative Design}

\author{
Afonso, J., \& Mesquita, I. \\ University of Porto, Faculty of Sport, Porto, Portugal, \\ Centre of Research, Education, Innovation and Intervention in Sport, Porto, Portugal, \\ Portugal
}

\section{Summary}

Contact:jneves@fade.up.pt

Developing decision-making abilities will enhance performance in team sports. However, few protocols exist for evaluating decision-making skills in live settings, and even fewer adopt representative designs. We present a four-step protocol for evaluating decision-making in defensive volleyball tasks, using a representative design with increasing complexity. Eye movement registration and verbal reports of thinking are used to capture relevant data. This protocol will provide information concerning the cues capturing the player's attention; hence, it will afford better individualization of the training processes for each player. Repeated application of this protocol will provide information on the players' progression and allow fine-tuning of the training process.

Key words: evaluation; decision-making; representative design; volleyball

\section{Introduction}

Research on decision-making has elicited a considerable interest in team sports (Roca et al., 2011). Notwithstanding, decision-making is usually left out of the evaluation protocols used in the training process. In volleyball, there is a paucity of investigation is this field; the works of Afonso et al. (2012), Piras, Lobietti and Squatrito (2010), and Moreno et al. (2008) stand out. Furthermore, research should develop representative designs, enabling the drawing of conclusions to real-life performance (Brunswik, 1955). Namely, the possibility to physically interact modifies the subject's anticipatory response, underlining the relevance of creating experimental conditions and tasks that reproduce, as reliably as possible, the natural context of performance (Bruce et al., 2012).

Therefore, the purpose of our work is to provide coaches and researchers in sports sciences with guidelines for elaborating experiments that scrutinize the processes underpinning decision making in team sports, while using a representative design. A set of representative, complex defensive tasks in volleyball will be used, while combining distinct methods for collecting information. 


\section{The Brunswik Society News/etter, 2013}

Implementing the protocol

Research on perceptive-decisional mechanisms underpinning performance in team sports has relied extensively on analyzing ocular behaviors to infer attentional factors involved in performance (Mann et al., 2011). Notwithstanding, it is known that point-of-gaze as well as duration may not correspond linearly to the locations from which information is being captured, nor to the quantity of information processed (Vickers, 2009). In this vein, analysis of verbal reports provides a window into the athlete's cognitive processes (McPherson \& Kernodle, 2007) and may be a sound complement for this type of research. The purpose of the current protocol is to examine the processes underpinning decision making in a defensive volleyball task, applying a representative design while combining simultaneous eye movement recording and immediate retrospective verbal reports of thinking.

The action will take place on a standard size volleyball court. In all four tasks, the players will assume a backcourt defensive position and attempt to defend attacks from the opposite team. Measures from eye movements will be registered in real-time, with the participants using an eye-tracking system. After each trial, the participants will leave the court to provide immediate retrospective verbal reports of thinking ('What were you thinking about while playing this point?'), following the protocol developed by McPherson (2000) and later adapted to the requirements of volleyball (Moreno et al., 2008).

In the first task, defender against single attacker (Figure 1), the coach tosses a ball to the setter, who sets the ball to the attacker. This task will provide information concerning how the defender analyzes the attacker; specifically, how the attacker moves, the direction of his run-up, trunk and arm actions, relation with the ball, power put into the preparatory phase of the action, among others.

In the second task - defender against setter and multiple attackers (Figure 2) the coach will toss the ball to the setter, but the team will have three attackers available. This second task will afford understanding on how the defender analyzes the setter's actions, namely his or her body language and position, in attempting to anticipate the attacker that will be served by the set. Furthermore, the defender will also have to analyze whether the attackers made any movements previous to the set that can enhance the chances of anticipating the attack location. 


\section{The Brunswik Society Newsletter, 2013}

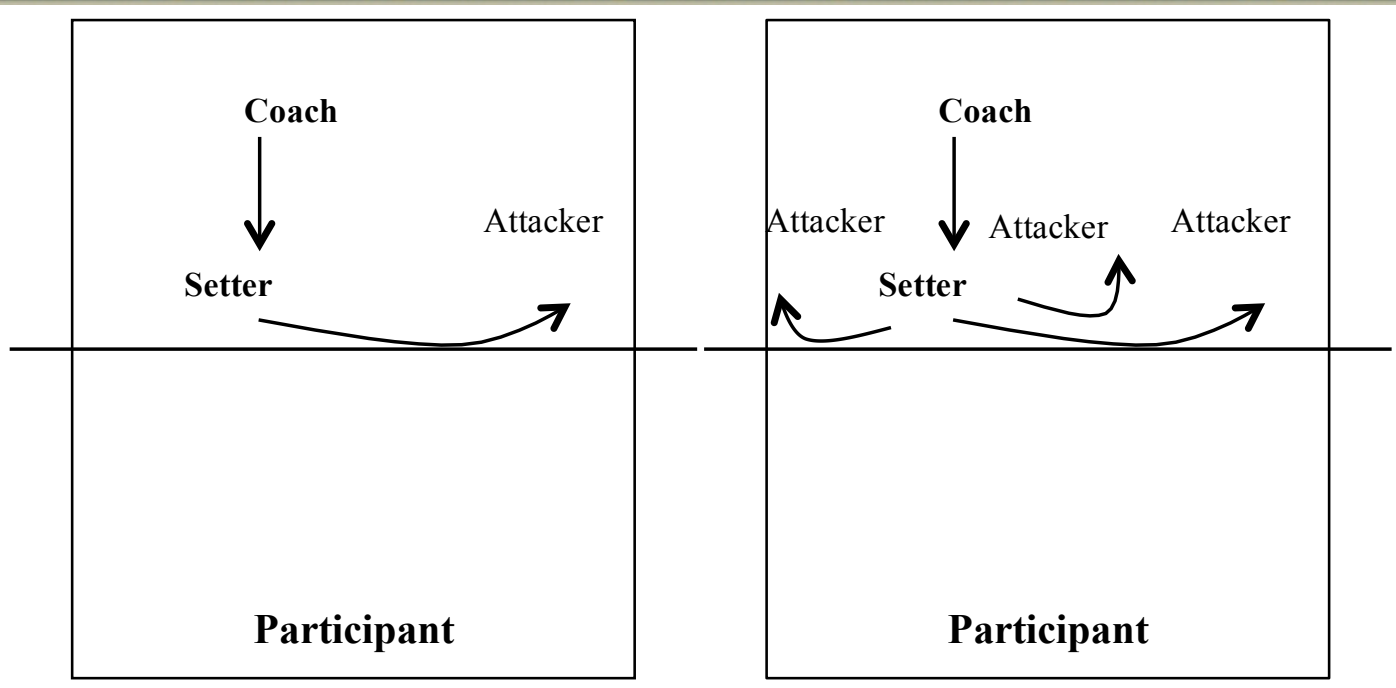

Figure 1. Defender against single attacker.

Figure 2. Defender against setter and multiple attackers.

Task number three - defender against setter and multiple attackers, with blockers opposing the attack (Figure 3 ) - will add the actions of the blockers of the defender's team. Besides analyzing the opponents, the defender must now act in coordination with his teammates, namely considering the zones covered by the block and the possibility of the ball being deflected by the blockers. This will also introduce a novel possibility for the attackers, as they can now explore the block in their advantage. Thus, the defender will have to consider this possibility and adapt its perceptive-decisional strategies accordingly.

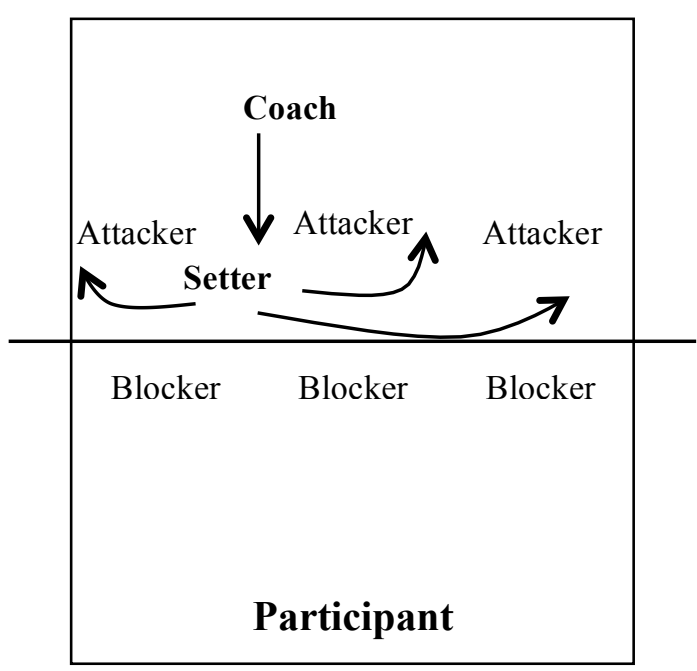

Figure 3. Defender against multiple opposition, with blockers.

In the fourth task, defender in full 6 vs. 6 scenarios, participants will attempt to defend attacks during 6 vs. 6 game situations. The sequences start with a serve towards the opposite side, after which the opposing team will run an attack sequence. The defending team will present three blockers and three backcourt defenders, one of 
which (zone 6) is the participant in the study. This task will generate the possibility of analyzing the quality of the first contact, which constrains the setter's options and also the attackers' movements before the set. Moreover, the defender's team will now be complete, and his defensive action will have to be coordinated not only with the blockers, but also with his two colleague defenders. As different possibilities for action emerge, perceptive-decisional strategies are expected to become more complex and specific.

\section{Applications}

It was our purpose to develop a representative experimental design that could assist coaches and researchers in better capturing the mechanisms underpinning perceptual-decisional expert performance, using a representative defensive task in volleyball to illustrate the core principles. A combination of verbal reports of thinking with eye-tracking data was applied for collecting data. The proposed protocol will afford useful information concerning the cues that capture the player's attention, and coaches can then analyze whether to reinforce or to change the player's strategies.

Overall, data from the four tasks may provide an indication of the tactical and technical level of the analyzed players and/or teams, thus affording the coach with powerful information to regulate the training process, specifically the complexity of the drills. It will also be possible to better individualize the training scenarios and the feedback given to each player, according to the nature of their performance in these tasks. Even for physical preparation, these tasks will contribute considerably, since capturing the most adequate information and knowing what to do with it will enhance the players' reaction times, improving their performance.

\section{References:}

Afonso, J., Garganta, J., McRobert, A., Williams, A. M., \& Mesquita, I. (2012). The perceptual cognitive processes underpinning skilled performance in volleyball: Evidence from eye-movements and verbal reports of thinking involving an in situ representative task. Journal of Sports Science and Medicine, 11, 339-345.

Bruce, L., Farrow, D., Raynor, A., \& Mann, D. (2012). But I can't pass that far! The influence of motor skill on decision making. Psychology of Sport and Exercise, 13, 152-161.

Brunswik, E. (1955). Representative design and probabilistic theory in a functional psychology. Psychological Review, 62, 193-217.

Mann, D., Coombes, S., Mousseau, M., \& Janelle, C. (2011). Quiet eye and the Bereitschaftspotential: Visuomotor mechanisms of expert motor performance. Cognitive Processing, 12, 223-234.

McPherson, S. (2000). Expert-novice differences in planning strategies during collegiate singles tennis competition. Journal of Sport and Exercise Psychology, 22, 39-62.

McPherson, S, \& Kernodle, M. (2007). Mapping two new points on the tennis expertise continuum: Tactical skills of adult advanced beginners and entry-level professionals during competition. Journal of Sports Sciences, 25, 945-959.

Moreno, M. P., Moreno, A., Ureña, A., Iglesias, D., \& Del Villar, F. (2008). Application of mentoring through reflection in female setters of the Spanish National Volleyball team: A case study. International Journal of Sport Psychology, 39, 59-76.

Piras, A., Lobietti, R., \& Squatrito, S. (2010). A study of saccadic eye movement dynamics in volleyball: Comparison between athletes and non-athletes. Journal of Sports Medicine and Physical Fitness, 50, 99-108.

Roca, A., Ford, P., McRobert, A., \& Williams, A. M. (2011). Identifying the processes underpinning anticipation and decision-making in a dynamic time-constrained-task. Cognitive Processing, 12, 301-310.

Vickers, J. (2009). Advances in coupling perception and action: The quiet eye as a bidirectional link between gaze, attention, and action. Progress in Brain Research, 174, 279-288. 


\title{
The Brunswik Society News/etter, 2013
}

Interests as a Component of Adult Course Preferences

\author{
James A. Athanasou \\ University of Technology Sydney \& Private Practice, \\ AUS
}

Contact: athanasou@gmail.com

This contribution to the Newsletter probably represents my last submission now that I have retired for some years and am quickly running out of steam. I hope that the reader will allow me some recollections before continuing with the substance of my contribution which for the most part relies on previous knowledge and little that is new.

My interest in Brunswik was stimulated in the early 1980s by Ray Cooksey who was my doctoral supervisor at the University of New England. My reading of the works in this field by many capable researchers provided me with a different outlook on psychology and one for which I am eternally grateful. Equally I am proud of my small involvement with the Brunswik Society, to which I had the honor to be introduced.

As a result, I now understand that for the most part group studies in psychology do not have generalizability and that the findings of most studies are not cumulative. I have come to respect the social judgment perspective in human behavior, and over time I have become enamored with the lens model.

Indeed, in the inside cover of my pocket diary, I carry three equations with me that I consider have enormous implications for behavioral science. These are: Hick's Law (information theoretic entropy); the Rasch equation; and the lens model equation. Probability is the common link. I wish that I had the ability to unravel this link.

My research career has been largely in the area of vocational interest assessment and only a few studies have been Brunswikian in focus. There is nothing ground-breaking in my publications; for the most part they are those of a journeyman. Nevertheless it has been immensely rewarding and enjoyable. For me, the highlight was the Brunswik conference at Landau. Anyway, from time to time I apply the Brunswik lens to the questions that I research.

The last paper (in press) which is the topic of this report to the Newsletter was a request (when you get academically older then people start to ask you for papers and book chapters). It focused on the role of creativity in course choices.

The role of interest in adult and vocational education as a mental resource for learning is well-known to educators. In teaching contexts, however, interest is considered mainly in terms of gaining student attention or enhancing educational curiosity, yet there are other aspects such as entrenched knowledge, emotional commitment, and relevant abilities that characterize a personal or longstanding interest. 
I believe that career interests share the same cognitive, conative and affective platform as educational interest. Vocational interests often act as the precursor for adult learning because they encompass the initial preference.

Notionally it is considered that for many learners a career interest should be a component of course choices. Naturally, individuals will differ and this study considered to what extent a person's individual educational choices are mediated by their career interests. I examined this through the medium of an intensive case study. The repeated choices of adults were examined in order to determine any pattern in their choices and to describe their decisions.

Figure 1 describes the model for investigating the individual judgments. The ellipses in Figure 1 represent items of information embedded in a course title. In this study the embedded features are the six vocational personality types formulated by Holland. Each educational course can be classified by these six types. For instance, accounting was classified as involving principally enterprising and conventional interests in the Holland model; art is exclusively artistic in the Holland model; computer technology involves a mixture of realistic, investigative and conventional interest types in the Holland model and so forth. As a person reacts positively or negatively to a course they may implicitly respond to these cues (i.e., implicit types). If we present enough choices then it is possible to describe the role (if any) of such career interests. The individual making the choices would not be aware, however, that they are responding to a series of coded implicit features.

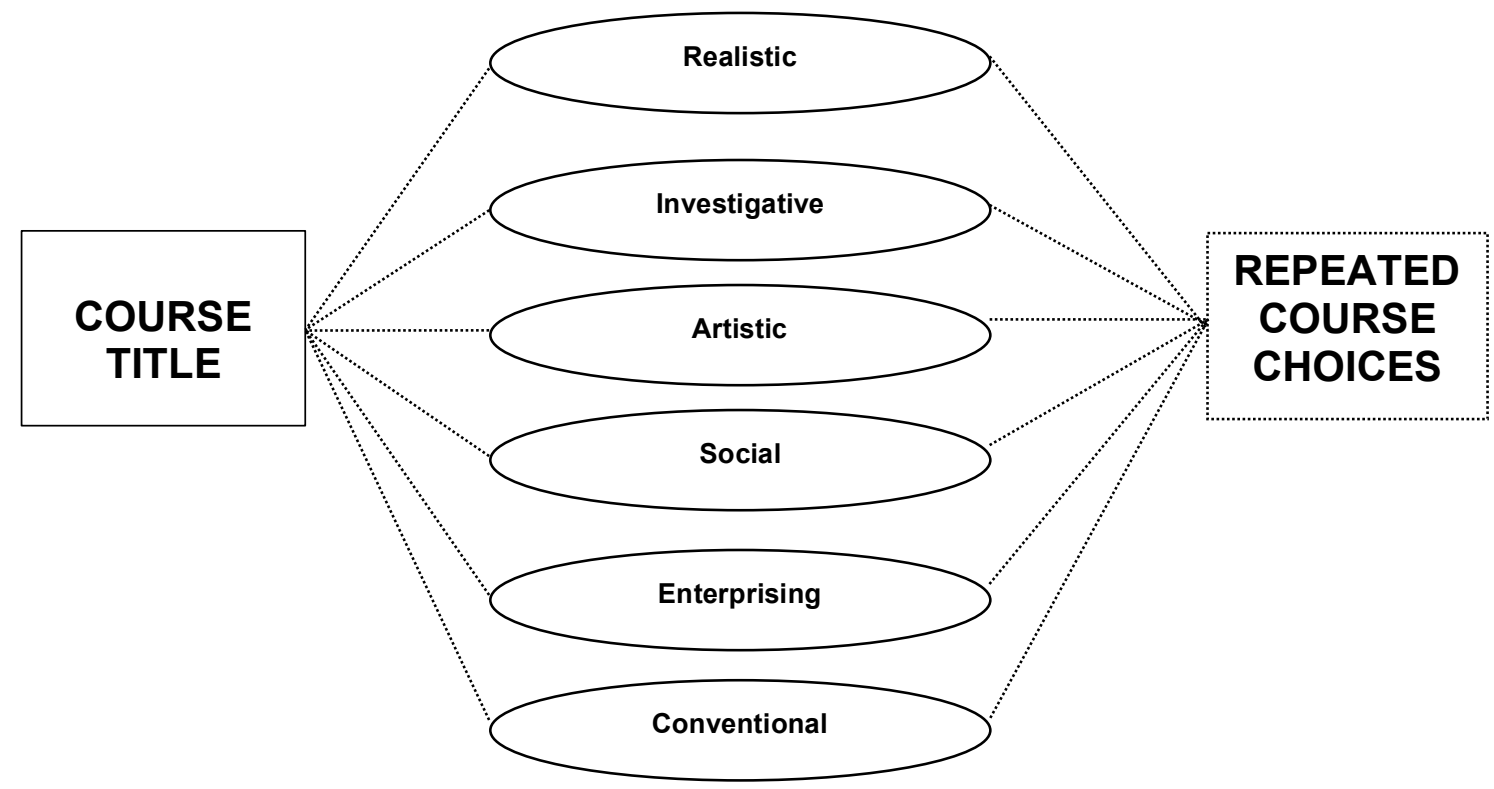

Figure 1. Implicit features that are present or absent in a course title.

In this study I presented adults with a range of courses that were advertised as available at a local community college and asked them to choose which ones they might be likely to apply for. It was hypothesized that each person would respond to implicit features of courses in lawful but idiosyncratic ways. 
Four adults (you do not get much time when journal editors are in a hurry and desperate) participated in an educational-vocational assessment and in the process of providing vocational guidance their course selection was examined using a logistic regression. The intra-rater consistency of choices ranged from $88 \%-100 \%$ for repeated course profiles. Choices varied from 2-11 courses. Results supported a minor role for vocational interest in these course choices. The overall R-squared values were around .281-.764. It was concluded that (a) adult choices did not rely exclusively upon interests; (b) adults used complex decision-making policies; and (c) the policies adopted were idiosyncratic.

Participants had some insight into their judgment policy. Nevertheless, it was not possible to model the judgments with a high degree of accuracy mainly because of necessity so many courses are rejected. My imprecise observation is that they took cognizance of their dislikes but then other factors such as relevance and value intervened in making the final choices.

Career interest may be necessary but still not sufficient for the course choices of many adults. Despite the value of relevance of one's interests, in adult education, they did not appear to affect course choices in any obvious way. Paradoxically negative interest (i.e., dislike) may be more useful in predicting course rejections.

The findings confirmed the huge individuality in the course choice process of adults. It would have been nice if community college course choices were based solely on interest but the world is a messy place. So, dear colleagues, this is probably ( $p>0.5)$ my Brunswik swansong. As David Adams of The Hitchhiker's Guide to the Galaxy might have said: "So long and thanks for the lens model"!

\section{Acknowledgement}

It would be remiss of me not to acknowledge the cooperation and support of Lars Sjödahl, Esther Kaufmann, Ray Cooksey, Ken Hammond and the late Bernard Wolf. 


\title{
The Brunswik Society News/etter, 2013
}

\section{News from the Past Year}

\author{
Jason W. Beckstead \\ University of South Florida College of Nursing, \\ US
}

Contact: jbeckste@health.usf.edu

This year I published results of a study of physicians that members of the Society may find interesting. An abbreviated abstract follows:

The benefits of prescribing cardiac rehabilitation (CR) for patients following heart surgery is well documented; however, physicians continue to underutilize CR programs and disparities in the referral of women are common. The research presented here employed clinical judgment analysis (CJA) to discover the tacit judgment and referral policies of individual physicians. The specific aims were to determine 1) what these policies were, 2) the degree of self-insight that individual physicians had into their own policies, and 3) the amount of agreement among physicians. Thirty-six Canadian physicians made judgments and decisions regarding 32 hypothetical cardiac patients, each described on five characteristics (gender, age, type of cardiovascular procedure, presence/absence of musculoskeletal pain, and degree of motivation). Consistent with previous studies, there was wide variation among physicians in their tacit and stated judgment policies and self-insight was modest. On the whole physicians showed evidence of systematic gender bias as they judged women as less likely than men to benefit from CR. Insight data suggest that one in three physicians were unaware of their bias. There was greater agreement among physicians in how they described their judgments (stated policies) than in how they actually made them (tacit policies). These findings offer some explanation for the slow progress of efforts to improve CR referrals and for gender disparities in referral rates.

Reference:

Beckstead, J. W., Pezzo, M. V., Beckie, T. M., Shahraki, F., Kentner, A. C., \& Grace, S. L. (2013). Physicians' tacit and stated policies for determining patient benefit and referral to cardiac rehabilitation. Medical Decision Making. doi:10.1177/0272989X13492017 


\title{
A Personal Journey in the Land of Brunswik and Colleagues
}

\author{
Rami Benbenishty \\ Bar-llan University, Ramat Gan, \\ Israel \\ Contact: ramibenben@gmail.com
}

My meeting with Brunswik was uneventful. I was walking down the stairs in the Paul Barewald School of Social Work at the Hebrew University of Jerusalem, when Monica Shapira asked me whether I would be interested in being her assistant, working with her on her Ph.D. thesis. The year was 1976 and Monica was a mythological figure - a holocaust survivor who worked with WW II refugees, and led the Rehabilitation Department of Israel's soldiers. She decided that her response to the Nazis was to come back to school and finish her doctorate. She was very fortunate to have the legendary Tversky and Kahneman as her thesis supervisors. They recognized how special and different she was and accepted her as a student, despite the fact that her line of research and methodology were far from their breakthrough work.

Monica introduced me to Brunswik, Hammond, Einhorn and the "Lens Model". Her dissertation was a statistical modeling of the decisions of probation officers on the disposition of juvenile delinquents, based on content analysis of their case files. My MA thesis was on a side issue - could we predict their decision, based on missing information; that is, if we know what they did not have recorded in their files. Interestingly, I could explain a very large proportion of the variance based on missing information.

This is when I fell in love with Egon. Despite the fact that no one in social work was interested in this line of work, I was captivated with the interplay between theory and reality, captured in the lens model and in discussions of simple models or simple processes. I conducted a long series of studies that had the lens model and Probabilistic Functionalism in the background. Unfortunately, in almost any area of social work I worked, the "ecological validity" could not be established. In most, if not all of my studies, I needed to focus on one side of the lens model, framing it as "policy capturing", describing and explicating underlying decision models. I was unable to examine satisfactorily the extent to which judgment and criterion were associated.

Since completing my thesis, I conducted many studies in several content areas and used several different methods. For several years I was a researcher at the Mental Health Department of the IDF. I studied the decision whether to discharge a soldier due to mental health difficulties. This was a very fruitful line of research, as it explicated a decision that was decentralized among many clinicians who never met and had only a vague idea why a soldier should be discharged (except for the extreme psychotic breakdowns that did not require any professional judgment). I mainly analyzed the content of case files. This study provided very important insights regarding the implicit policy underlying the decision - mainly, discharge did not reflect 


\section{The Brunswik Society

"mental health" issues, as it was depicted, but judgments of potential to adjust to military life, and the potential difficulties the military may experience with disciplining the soldier. I hope and believe that my presentations of these insights were instrumental to bring about discussions on this issue and eventually policy changes (Benbenishty, Zirlin-Shemesh, \& Kaplan, 1993).

The work on discharge led to my interest in developing decision aids based on integrating insights from several methods, including statistical modeling a la judgment analysis and "think aloud" in order to identify rare cases that could not be captured statistically (Benbenishty, Zirlin-Shemesh, \& Kaplan, 1993). In an interesting twist I created a computer program that required a judge to actively "ask" for information on a case. The program had an underlying data base extracted from case files. The participants asked for all the information they needed about the case and after examining it made decisions whether to discharge the soldier. I could then conduct two related lines of analysis - compare the real life decisions with those based on the computer representation and identify patterns of information search. One of the very interesting insights was that expert decision makers may have used different information search patterns (e.g., start with the present and go back in time, or the other way around) but agreed on the decision. A central interpretation was related to one of Brunswik's ideas that cues in the environment are interrelated and redundant, and therefore wherever you looked into the lives of these soldiers you saw the same underlying picture.

Insights were also gained when we explored issues of "aggregated judge". When I compared the decision models extracted from decisions made in two different military bases we found that these models differed in nontrivial ways. It raised many interesting issues on whether these models reflect two different ecologies (i.e., soldiers and circumstances were very different), or two different cognitive models (e.g., different disciplines and theoretical approaches).

This led to the idea of exploring individual models; that is, instead of aggregating all judges in a setting and trying to model their shared model, I decided to examine whether there are discrete individual models, and perhaps a limited set of such individual models. Shapira and I conducted the study in the setting of child welfare, an area I have been studying ever since. In this study we created a set of 120 stimulus cases that could be presented to each of the judges in order to estimate an individual model. In contrast to the common practice of manipulating case factors in an orthogonal design, we tried to follow Brunswik's idea of "ecological representativeness" in the structure of correlations among the case factors. We used a computer program that generated a large set of case profiles (10 case characteristics); the underlying matrix of correlations was provided by us, trying to introduce inter-item correlations that seemed to reflect what we know about these relationships in reality. We then had professionals review and judge (judgment of risk and decision on case disposition) a sample of case profiles (Shapira \& Benbenishty, 1993).

Since this study I have carried out a large number of studies using the lens model, and worked with a rather large number of masters students who have conducted their empirical theses on a wide range of decisions in professional settings, explicating current practices. Most, if not all, were based on content analyses of case files. 


\section{The Brunswik Society News/etter, 2013}

In the last decade or so I carried out studies in the context of the decision made by an interdisciplinary hospital team that needs to assess whether a hospitalized child is at risk for abuse and neglect and whether the case should be reported to the child protection services. I started with studies that analyzed existing files (Arad-Davidson \& Benbenishty, 2008; Benbenishty \& Chen, 2003), but recently I was able to conduct a study in which practitioners completed detailed questionnaires on each of the almost 1000 cases investigated (Benbenishty et al., in press). This study also enabled us to move one step further and ask: what happened to the cases that were reported to protective services; how many of them were actually substantiated? This is as close I got to ask about accuracy of judgment. However, it is limited because due to ethical considerations we were not allowed to follow up on cases that were not reported. This of course eliminates the possibility of estimating the statistical relationship between the practitioners' judgment and the criterion. Nevertheless, were able to assess how many reports made by hospital staff were seen as "mistaken" (very few) and what characterizes the cases that upon further investigation of protective service workers in the community was substantiated as a case of abuse.

Finally, my years of working with Bilaha Arad Davidson on decision making in child welfare suggested that Brunswik's work, and even the work of Hammond, have, for my work, a major limitation in neglecting the larger context in which decisions are being made. Our work in international comparisons of decisions strongly suggests that the same cases are being assessed differently in different countries, but there is an even stronger effect - decisions on the "same" cases (vignette stimuli) are different in different contexts. I do not think that adding an interaction term to the equations to reflect a different context solves the theoretical difficulty. While Brunswik was addressing basic human perception and Hammond's work, to my (limited) knowledge, did not have context as a major consideration, there seems to be a need to find ways of examining real life decisions, in professional areas such as child welfare, with context as an integral part of the model. In recent years I have coordinated an international study that used vignettes and asked practitioners and students to make judgments and recommendation of case dispositions. There were clear differences between countries in responses to the same vignettes. This suggests that the context of their protective systems had an impact on their decisions.

It will be great to find colleagues who know and understand Brunswik's theory, to explore the ways contexts can be integrated into our understanding of human decision making.

References and further information:

Arad-Davidson, B., \& Benbenishty, R. (2008). The role of worker's attitudes and parent and child wishes in child protection workers' assessments and recommendation regarding removal and reunification. Children and Youth Services Review, 30(1), 107-121.

Arad-Davidson, B., \& Benbenishty, R. (2009). Contribution of child protection workers' attitudes to their risk assessments and intervention recommendations: A study in Israel. Health \& Social Care in the Community, 18(1), 1-9.

Benbenishty, R. (1992). An overview of methods to elicit and model expert clinical judgment and decision making. Social Service Review, 66(4), 598-616.

Benbenishty, R., \& Chen, W. (2003). Decision-making by the Child Protection Team of a medical center. Health \& Social Work, 28(4), $284-292$.

Benbenishty, R., Davidson-Arad, B., Chen, W., Glasser, S., Tzur, S., \& Lerner-Geva, L. (2011). The decision of hospital-based child protection teams to report to community child protective services. British Journal of Social Work, 41,1232-1250. 
Benbenishty, R., Jedweb, M., Chen, C., Glasser, S., Slutzky, H., Siegal, G., Lavi-Sahar, Z., LernerGeva, L. (in press). Predicting the decisions of hospital based child protection teams to report to child protective services, police and community welfare services. Child Abuse \& Neglect.

Benbenishty, R., Osmo, R., \& Gold, N. (2003). Rationales provided for risk assessments and for recommended interventions: A comparison between Canadian and Israeli professionals. British Journal of Social Work, 33(2), 137-155.

Benbenishty, R., \& Treistman, R. (1998). The development and evaluation of a hybrid decision support system for clinical decision making: The case of discharge from the military. Social Work Research, 22(4), 195-204.

Benbenishty, R., Zirlin-Shemesh, N., \& Kaplan, Z. (1993). Policy capturing: Discharge from the Israeli army due to mental difficulties. Military Psychology, 5(3), 159-172.

Gold, N., Benbenishty, R., \& Osmo, R. (2001). A comparative study of risk assessments and recommended interventions in Canada and Israel. Child Abuse \& Neglect , 25(5), 607-622.

Shapira, M., \& Benbenishty, R. (1993). Modeling judgments and decisions in cases of alleged child abuse and neglect. Social Work Research and Abstracts, 29(2), 14-19.

\title{
Construct-irrelevant Mental Processes in University Students' Responding to Business and Economic Test Items: Using Symmetry Based on Verbal Reports to Establish the Validity of Test Score Interpretations
}

\author{
Sebastian Brückner \\ Johannes Gutenberg-University Mainz, \\ GER
}

Contact: brueckner@uni-mainz.de

Construct-irrelevant mental processes, such as the use of test taking strategies like guessing or testwiseness, often affect the validity of test score interpretations from standardized educational tests (e.g., Multiple Choice Tests) in a negative way (Kane, 2013; Rebeck \& Asarta, 2012). However, most studies in the domain of business and economics using standardized tests have focused on the modeling of constructrelevant mental processes (e.g., Vernooij, 1995) and neglected construct-irrelevant mental processes. Therefore, they must be considered more thoroughly in the analysis of mental processes during validation (see Borsboom, Mellenbergh, \& van Heerden, 2004; Leighton, Heffernan, Cor, Gokiert, \& Cui, 2011). The analysis of mental processes strives to compare the expected processes, modeled by the test developers, with the actually observed processes, displayed by the subjects. More specifically, mental processes are postulated based on assumptions about the knowledge and skills of purposefully selected samples (criterion) and are then validated by comparison with the processes verbalized by the subjects in verbal reports (predictor) (see Leighton et al., 2011). To support validity, the use of compensatory test taking strategies (like guessing or eliminating) would need to vary according to the level of knowledge that students have acquired over the course of their studies (Hamdan, 1979). Accordingly, subjects with low-level business and economic expertise (e.g., students from another field of study) are expected to use more compensatory test taking strategies in an economics test, whereas subjects with high-level expertise (e.g., students from business and economics degree courses) are expected to use fewer such strategies. 


\section{The Brunswik Society Newsletter, 2013}

If there is a perfect alignment between the expected and observed mental processes, it can also be described as symmetry - with reference to Wittmann (1990; 1988) and Brunswik (1955). If the expected processes align with the observed processes, their symmetry supports validity; accordingly, asymmetry of the processes compromises validity. Hence, this analysis of mental processes bears clear similarities with Brunswik's symmetry and his "lens model" (Brunswik, 1955, p. 206), which can function as a framework for the analysis. Like Brunswik's symmetry, the analysis of verbal reports can point to different kinds of symmetries or asymmetries if the final choice of a respondent is taken into consideration. For example, it is possible that an expert guesses (1st-level misalignment); even though he or she is expected to mainly use construct-relevant knowledge and skills. Instead, the expert uses constructirrelevant mental processes and may select a wrong answer, which would correspond with common guessing behavior and would undermine a valid test score interpretation (2nd-level misalignment). Furthermore, it is possible that there are several inconsistencies in the response behavior, for example, if the expert guesses, but nevertheless selects the correct answer, which would be an inconsistent outcome for common guessing behavior (3rd-level misalignment). While this case might of course support test score interpretations based on aggregated test scores, it would generally put into question a valid assessment of a given construct. Borsboom et al. (2004) and Wittmann (1988, p. 539) are in agreement that a "high correlation coefficient can be taken as a sign of high [predictive] validity, but it is only a necessary yet insufficient condition for high construct validity", especially since "[...] we see how misleading correlation coefficients can be, if we do not know what units they represent".

In my study, two groups of students with different degrees of expertise are asked to think aloud while responding to 20 business and economic test items in closed-ended format. ${ }^{1}$ The first group consists of students from business and economics degree courses, who are considered experts, while the second group consists of students of other social scientific disciplines unrelated to business and economics, who are considered lay people. The latter are expected to have a general understanding of business and economic issues, but do not have professionally developed subject-specific mental structures and are therefore expected to use mainly construct-irrelevant mental processes when responding to business and economic test items. To analyze this issue more closely and to evaluate its use for the validation of item score interpretations, three hypotheses are formulated and tested in the following.

Students use more compensatory test taking strategies when responding to items with high psychometric difficulty than when responding to items with low psychometric difficulty.

Both students of business and economics and students of other social scientific disciplines unrelated to business and economics give more incorrect answers when responding based on compensatory test taking strategies rather than on knowledge.

Students of business and economics use fewer compensatory test taking strategies than students of other social scientific disciplines unrelated to business and economics.

\footnotetext{
${ }^{1}$ Altogether, the sample included data from $\mathrm{N}=120$ item responses. The items came from the WiwiKom project (see Zlatkin-Troitschanskaia et al., 2013) or wiwi-kompetenz.de.
} 


\section{The Brunswik Society News/etter, 2013}

The verbal reports were inductively coded multiple times and finally three codes were differentiated ("guess" = no sufficient mental representation, "elimination" = incomplete mental representation, and "knowledge" = complete mental representation) to characterize the mental processes. To ensure reliability of the coding scheme, $35 \%$ of the data were coded again by a second, independent coder and Krippendorff's $\alpha$ (Hayes \& Krippendorff, 2007) was computed. Since this was a primarily exploratory analysis the moderate inter-rater agreement $(\alpha=0.7513)$ passed the required coefficient level ( $\alpha=0.667$ in Krippendorff, 2004).

With regard to the first question, whether more difficult items (marked by a low difficulty index) prompted the students to guess more, a variance analysis was conducted comparing the average item difficulty from the total sample of the first WiwiKom survey $(\mathrm{N}=3783)$ to the response behavior in the verbal reports. The analysis indicated, with a medium effect size, that subjects who used a guessing strategy did guess more when responding to items with higher item difficulty ( $\mathrm{F}=$ $\left.6.406, \rho<0.01, \eta_{\mathrm{p}}^{2}=0.103\right)$. With regard to the second question, whether guessing and elimination led to more incorrect answers, a chi-square independence test was conducted and Cohen's w (Cohen, 1988) was used as a measure of effect size. The test indicated, with a high significance, that the two variables (use of compensatory test taking strategy and correct answer) are not independent. On the whole, random guessing led rather to a wrong answer. For systematic elimination, the percentage of correct answers was about $40 \%$. The subjects who did not use guessing or elimination gave only four incorrect answers out of 120 responses. This was confirmed with a large effect $\left(\chi^{2}=52.444, \rho<0.001, w=0.661\right)$. The findings were the same for the specific sub-groups. For both students of business and economic subjects $\left(\chi^{2}=\right.$ $19.415, \rho<0.001, w=0.569)$ and students of social sciences $\left(\chi^{2}=31.011, \rho<\right.$ $0.001, w=0.719$ ), the findings were highly significant with large effect sizes. Furthermore, the results to the third question showed that students of business and economics did not need to guess as often as students of other social scientific disciplines. The students of business and economics answered about two thirds of the items based on a substantial mental representation, whereas students of other social sciences knew the answers of only one third of the items. The results are statistically significant with a medium effect size $\left(\chi^{2}=12.015, \rho<0.01, w=0.316\right)$.

To sum up, the analyses provided preliminary evidence on the validity of the item score interpretations. Particularly the test taking strategy of guessing was used according to expectations primarily by the sub-group of students of social sciences, and it yielded an accordingly smaller number of correct answers. However, in further studies much more research efforts ought to be focused on the different types of testing strategies - especially the "elimination" strategy. It would be quite possible that both groups use this strategy in different ways. One group, having common knowledge of business and economics, might tend to ignore some aspects of an item, while the other group, having developed professional subject-specific knowledge structures, might be able to eliminate some aspects and might thus select a correct answer more often. The detailed analysis of this question should also take into account the business and economics students' previous knowledge from an apprenticeship or a part-time job and their class work. Another related question is whether the subjects develop different mental representations with regard to the item format or the content areas, and if they use test taking strategies differently. Furthermore, the use of test taking strategies is only one aspect of the complex mental processes taking place during item 
responding. Subsequent analyses should therefore also focus on construct-relevant mental processes as well as on further affective and meta-cognitive aspects. Only this way, can item score interpretations be explained in content and also be used for analyses of the symmetry of generalizable test score interpretations. In general, it can be noticed that lots of research is still needed to ensure extensive validity of test score interpretations based on mental processes. But in conclusion, this study showed that Brunswik's symmetry (Wittmann, 1990), which is already a proven method in psychological research, is also generally relevant on the item level for analyses of mental processes, and it can productively complement analyses on the symmetry of factorial and dimensional structures based on aggregated item scores.

\section{References:}

Borsboom, D., Mellenbergh, G. J., \& van Heerden, J. (2004). The concept of validity. Psychological Review, 111(4), 1061-1071.

Brunswik, E. (1955). Representative Design and probabilistic theory in a functional psychology. Psychological Reviews, 62, 193-217.

Cohen, J. (1988). Statistical Power Analysis for the Behavioral Sciences (2., revised Ed.). Hillsdale, NY: Lawrence Erlbaum Associates.

Hamdan, M. A. (1979). On the correction for guessing on a multiple-choice examination. The Journal of Experimental Education, 48(1), 29-31.

Hayes, A. F., \& Krippendorff, K. (2007). Answering the call for a standard reliability measure for coding data. Communication Methods and Measures, 1,77-89.

Kane, M. T. (2013). Validating the interpretations and uses of test scores. Journal of Educational Measurement, 50(1), 1-73.

Krippendorff, K. (2004). Reliability in content analysis: Some common misconceptions and recommendations. Human Communication Research, 30(3), 411-433.

Leighton, J. P., Heffernan, C., Cor, M. K., Gokiert, R. J., \& Cui, Y. (2011). An experimental test of student verbal reports and teacher evaluations as a source of validity evidence for test development. Applied Measurement in Education, 24(4), 324-348.

Rebeck, K., \& Asarta, C. (2012). Methods of assessment in the college economics course. In G. M. Hoyt \& K. M. McGoldrick (Eds.). International Handbook on Teaching and Learning Economics (pp. 177-187). Cheltenham, UK: Edward Elgar Publishing.

Vernooij, A. T. J. (1995). Problem Solving Strategies. In W. H. Gijselaers, D. T. Tempelaar, P. K. Keizer, J. M. Blommaert, E. M. Bernard \& H. Kasper (Eds.), Educational innovation in economics and business administration. The case of problem-based learning (pp. 69-77). Dordrecht: Kluwer.

Wittmann, W. W. (1990). Brunswik-Symmetrie und die Konzeption der Fünf-Datenboxen. Ein Rahmenkonzept für umfassende Evaluationsforschung. [Brunswik symmetry and the concept of the five data boxes. A framework for comprehensive evaluation research]. Zeitschrift für Pädagogische Psychologie, 4, 241-251.

Wittmann, W. W. (1988). Multivariate reliability theory: Principles of symmetry and successful validation strategies. In J. R. Nesselroade \& R. B. Cattell (Eds.), Handbook of multivariate experimental psychology, $2^{\text {nd }}$ ed. (pp. 505-560). New York: Plenum Press.

Zlatkin-Troitschanskaia, O., Förster, M., Brückner, S., Hansen, M., \& Happ, R. (2013, in press). Modellierung und Erfassung der wirtschaftswissenschaftlichen Fachkompetenz bei Studierenden im deutschen Hochschulbereich [Modeling and measuring competencies in business and economics among students and graduates in German Higher Education]. Lehrerbildung auf dem Prüfstand. 


\title{
The Brunswik Society News/etter, 2013
}

\section{The Cognitive Continuum and More on Bounded Rationality}

\author{
Mandeep K. Dhami \\ Middlesex University, \\ UK
}

Contact:m.dhami@mdx.ac.uk

Over the past couple of decades, dual process (systems) accounts of human cognition have preoccupied cognitive theorists and researchers, including those working in the JDM field. Much has been said about the pros of analytic thinking and the cons of intuition (with the notable exception of Gigerenzer's work). But, for Brunswik, Hammond, and some others (including myself), there is a large middleground between intuition and analysis called "quasi-rationality" (or sometimes common sense). Hammond $(1996,2000)$ formalised this in his "cognitive continuum theory." In addition to highlighting the multi-modal nature of cognition, Hammond points to the idea that the mode of cognition applied in a task is induced by the task environment itself; hence making it important for psychologists to study the environment. Finally, Hammond reminds us that the correspondence between the task and cognition represents the upper limit on performance; meaning that pure analysis will not always lead to the best performance. Although some dual process theorists (e.g., Sloman and Kahneman) do acknowledge the interplay between intuition and analysis, they have largely stuck to the extremes of human cognitive behavior. Perhaps this is not surprising. But, what is surprising is that Brunswikians appear to have simply ignored the cognitive continuum (with the notable exception of some early work by Hamm and by Dunwoody). Quasi-rationality is more characteristic of our thinking than the use of pure intuition and analysis alone, and yet this concept remains to be precisely defined and systematically investigated. In a recent paper, my colleague Mary Thomson (now at Northumbria University) and I reviewed the main tenets of cognitive continuum theory as well as the empirical work testing this theory, and explored the usefulness of quasi-rational thinking in the management domain (see abstract below).

The notion of bounded rationality is central to studying and understanding human cognition and behavior. Some of us investigating the cognitive processing models that might describe and predict behavior have suggested that noncompensatory models are a good candidate for understanding the nature of bounded rationality. In the past, I have demonstrated in the legal, criminal and medical domains, that non-compensatory models of judgment and choice outperform compensatory ones. Recently, I have also found that non-compensatory models are better than compensatory models (i.e., the subjective expected utility model) at predicting people's intentions to engage in a whole variety of risky behaviors. I have explored this topic with David Mandel (York University, Canada and DRDC-Toronto).

Below are abstracts for these recent papers: 


\section{The Brunswik Society Newsletter, 2013}

Dhami, M. K., \& Thomson, M. (2012). On the relevance of cognitive continuum theory for understanding management judgment and decision making. European Management Journal, 30, 316-326.

"Quasirationality" (i.e., the combination of intuitive and analytic thought) is increasingly considered to be widespread and beneficial in management. This paper provides an overview of this concept as it is defined by Cognitive Continuum Theory (Hammond, 1996, 2000), and highlights the relevance of the theory for studying managerial judgment and decision making. According to Cognitive Continuum Theory, there are multiple modes of cognition that lie on a continuum between intuition and analysis. Quasirationality is the prevalent mode of cognition. Cognitive (managerial) tasks vary in their ability to induce intuition, quasirationality or analysis, and performance is contingent on the correspondence between task properties and cognitive mode. Using Cognitive Continuum Theory, management researchers can identify tasks requiring different modes of thought, and recognize when quasirationality may outperform analysis and intuition. Researchers can also utilize Cognitive Continuum Theory to iron out some identified anomalies in the strategic management literature and to provide a more refined theoretical framework in this context.

Dhami, M. K., \& Mandel, D. R. (2012). Crime as risk taking. Psychology, Crime and Law, 18, 389-403.

Engagement in criminal activity may be viewed as risk-taking behavior as it has both benefits and drawbacks that are probabilistic. In two studies, we examined how individuals' risk perceptions can inform our understanding of their intentions to engage in criminal activity. Study 1 measured youths' perceptions of the value and probability of the benefits and drawbacks of engaging in three common crimes (i.e., shoplifting, forgery, and buying illegal drugs), and examined how well these perceptions predicted youths' forecasted engagement in these crimes, controlling for their past engagement. We found that intentions to engage in criminal activity were best predicted by the perceived value of the benefits that may be obtained, irrespective of their probabilities or the drawbacks that may also be incurred. Study 2 specified the benefit and drawback that youth thought about and examined another crime (i.e., drinking and driving). The findings of Study 1 were replicated under these conditions. The present research supports a limited rationality perspective on criminal intentions, and can have implications for crime prevention/intervention strategies.

Dhami, M. K., \& Mandel, D. R. (2012). Forecasted risk taking in youth: Evidence for a bounded-rationality perspective. Synthese, 189, 161-171. doi:10.1007/s11229012-0110-2

This research examined whether youth's forecasted risk taking is best predicted by a compensatory (namely, subjective expected utility) or non-compensatory (e.g., single-factor) model. Ninety youth assessed the importance of perceived benefits, importance of perceived drawbacks, subjective probability of benefits, and subjective probability of drawbacks for 16 risky behaviors clustered evenly into recreational and health/safety domains. In both domains, there was strong support for a noncompensatory model in which only the perceived importance of the benefits of engaging in a risky behavior predicted youths' forecasted engagement in risky behavior. The study overcomes earlier methodological weaknesses by fully decomposing participants' assessments into importance and probability aspects for 


\title{
The Brunswik Society News/etter, 2013
}

both benefits and drawbacks. As such, the findings provide clear evidence in support of a bounded rationality perspective on youth decision making regarding risk taking.

\section{Summary of Toward a Characterization of Adaptive Systems: A Framework for Researchers and System Designers}

\author{
Karen Feigh \\ School of Aerospace and Engineering, Georgia Tech, \\ US
}

Contact: karen.feigh@aerospace.gatech.edu

This article summarizes the Feigh et al. (2012) paper on the creation of a framework for adaptive systems for researchers and system designers. Adaptive systems are the technological component of joint human-machine systems that can change their behavior to meet the changing needs of their users, often without explicit instructions from their users. The goal of this paper is to present a systematic framework to characterize adaptive systems. The appeal of adaptive systems is that they can modify their behavior to better meet an operator's needs. Adaptive systems track and sense information about their users, their current tasks, and their environment to inform their adaptations. The concept of adaptive automation is appealing because it could create the capability of creating computer assistants that behave like good human assistants who can provide what is needed without being asked. However, the majority of adaptive systems have been experimental rather than practical because of the significant technical challenges in accurately perceiving and interpreting users' current cognitive state; integrating cognitive state, environment, and task information; and using it to predict users' current needs. Nonetheless, recent developments in sensors to identify users' cognitive state will undoubtedly increase interest and research in adaptive systems over the next few years.

This paper provides an organizing framework for characterizing adaptive systems, identifying considerations and implications, and suggesting future research issues. The framework has two parts that (a) categorize ways in which adaptive systems can modify their behavior and (b) characterize trigger mechanisms through which adaptive systems can sense the current situation and choose an appropriate adaption.

The framework presented here expands existing perspectives on what adaptive systems are. Previous descriptions of adaptive systems had a tendency to focus on only one type of adaptation: modifications of the allocation of functions performed by the user and the adaptive system. This framework acknowledges that there are many other ways in which adaptive systems can adapt; for example, by changing the amount of detail presented to users (which can be important when they are very stressed) or by changing the sensory modality in which information is presented: visual versus auditory. For each category, the paper provides technical descriptions, reviews the implications and considerations, and provides concrete examples drawn from the 


\section{The Brunswik Society

literature. The paper also discusses past and current challenges in creating successful adaptive systems, recent work to overcome those challenges, and future research directions to make adaptive systems more practical, usable, and reliable.

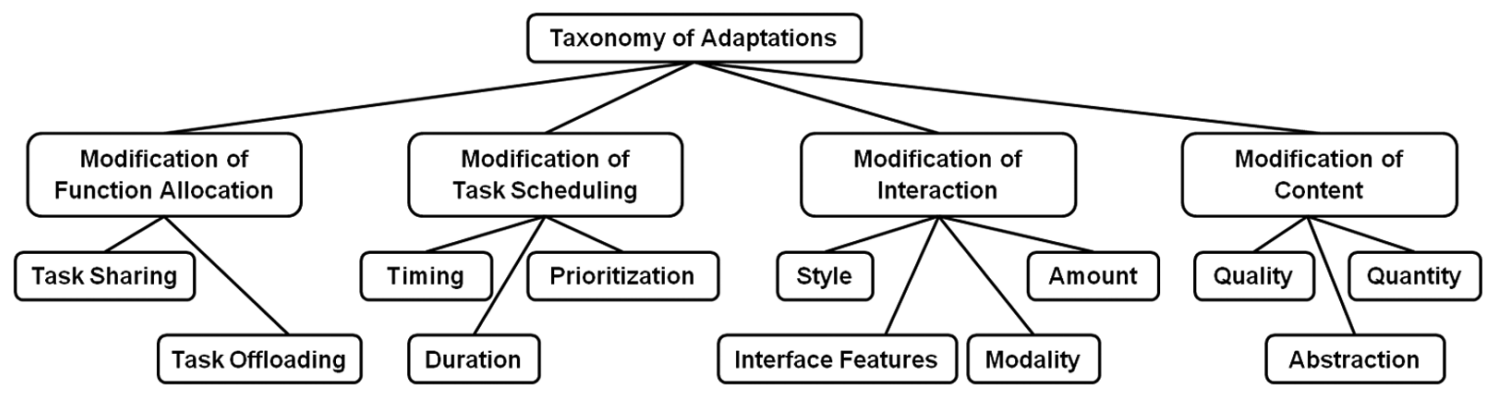

Figure 1. Taxonomy of Adaptations.

Figure 1 illustrates the Taxonomy of Adaptations for human-machine systems. The categories in the first row span the adaptation space and illustrate the four primary ways in which a designer might make the automated portion of a human-machine system adaptive to adjust to changing needs of the current situation:

Modification of function allocation. Dynamically changing who (human or machine) performs each function, task, or subtask. An example of this adaptation is an automatic breaking system on an automobile; human reflexes are sometimes too slow to avoid the crash if the other vehicles or obstacles are very close when it is first detected.

Modification of task scheduling. Dynamically changing when tasks are performed, including their duration and priority. Smart phones that change the ring tone to silent or vibrate when the calendar on the phone indicates that the operator has a scheduled activity is an example of this type of adaptation.

Modification of interaction. Dynamically changing how an automated system interacts with the users. Examples include changing the layout of a visual interface, the mode in which information is presented and received (e.g., visual, auditory, haptic), whether information is exchanged synchronously or asynchronously, and whether information is pushed or pulled. For example, the Communications Scheduler (Dorneich et al., 2010) adapts soldiers' communications during high workload times and changes the interaction so that instead of pushing information to soldiers as it comes in, the soldier must pull information from the system when workload allows.

Modification of content. Dynamically changing what information it presents to the user, including what level of detail or abstraction and categories of information are presented. For example, a map display in a car's navigation system may change the information content it displays by showing detailed information when traveling slowly though an urban area and a larger view when traveling at highway speeds through a rural area.

Although the top-level categories are mutually exclusive, adaptations in one category are likely to be used in combination with adaptations in another. 


\section{The Brunswik Society Newsletter, 2013}

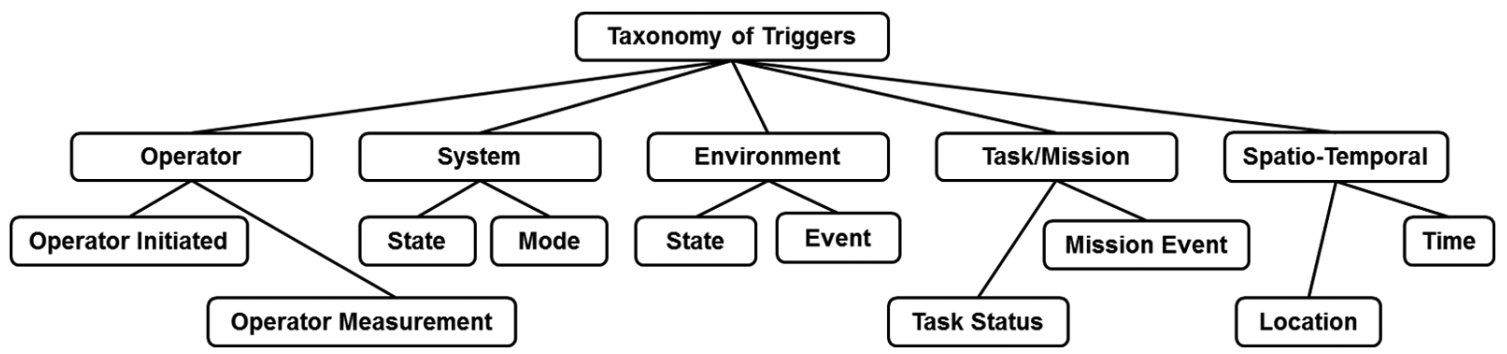

Figure 2. Taxonomy of Triggers for Adaptive Systems.

Adaptations are designed to support specific situations or contexts and accordingly, changes in context can trigger the system to adapt. Figure 2 illustrates the taxonomy of triggers. Triggers are based on several classes of information that can be sensed, observed, or modeled to create an understanding of context or "what is happening in the world" relevant to the adaptive system's programming. Triggers are used to identify when to engage an adaptation, how long an adaptation should persist, and when to disengage the adaptation. Prior work on adaptation management has focused principally on engagement triggers and neglected duration and disengagement criterion. The importance of disengagement triggers has been highlighted with aviation accidents caused by unanticipated autopilot disengagement (Billings, 1996). Expanding on previous categorizations (Byrne \& Parasuraman, 1996; Parasuraman, Mouloua, \& Molloy, 1996; Rouse, 1988; Sheridan \& Parasuraman, 2006), this taxonomy classifies adaptation triggers into five broad categories:

Operator-based triggers. Adaptations can be triggered by the operator directly or by a system assessment of the operator state.

System-based triggers. Current or predicted states of the system can be used to trigger adaptations. Different modes of system operations can also trigger adaptations.

Environment-based triggers. States of the environment or events external to the operator and the system can be used to trigger adaptations.

Task- and mission-based triggers. A mission is typically composed of a coherent set of goals and subgoals and accomplished by a set of tasks. Triggers can be based on task state or mission state.

Spatiotemporal triggers. Both time and location can be used as adaptation triggers.

The drive to design effective adaptive systems stems from a desire to support the changing needs of operators in complex, dynamic environments. Research into adaptive systems is a direct response to the need to provide multiple automation configurations that can be invoked based on the automation's assessment of specific contextual features - a move from point design to robust design. Challenges to successful implementation of adaptive systems include the difficulty of unobtrusively sense and interpret the user's cognitive state and implementing and controlling automation that can adapt itself. Recent improvements in sensors and classifiers, however, have made adaptive systems more practicable and encouraged new applications and additional research. The framework presented in this article provides a structure to help researchers to organize a diverse set of literature on adaptive 


\title{
The Brunswik Society

systems and help systems designers systematically consider the range of adaptations and triggers available.

\section{References:}

Billings, C. E. (1996). Aviation automation: The search for a human centered approach. Lawrence Erlbaum Associates, Hillsdale, N.J.

Byrne, E. A., \& Parasuraman, R. (1996). Psychophysiology and adaptive automation. Biological Psychology, 42, 249-268.

Dorneich, M. C., Mathan, S., Whitlow, S. D., \& Ververs, P. M. (2010). Etiquette considerations for adaptive systems that interrupt: Cost and benefits. In Hayes, C. C., \& Miller, C., (Eds.), HumanComputer Etiquette: Understanding the Impact of Human Culture and Expectations on the Use and Effectiveness of Computers and Technology. Taylor \& Francis.

Feigh, K. M., Dorneich, M. C., \& Hayes, C. C. (2012). Towards a characterization of adaptive systems: A framework for researchers and system designers. Human Factors, 54(6), 1008-1024.

Parasuraman, R., Mouloua, M., \& Molloy, R. (1996). Effects of adaptive task allocation on monitoring of automated systems. Human Factors, 38(4), 665-579.

Rouse, W. (1988). Adaptive interfaces for human/computer control. Human Factors, 30, 431-488.

Sheridan, T. B., \& Parasuraman, R. (2006). Human-automation interaction. Reviews of Human Factors and Ergonomics, 1, 89-129.

\section{Researching Expert Decision Making to Develop Online Training for Novices}

\author{
Priscilla Harries \\ Brunel University, \\ UK
}

Contact: Priscilla.Harries@brunel.ac.uk

My evolving program of research into expert decision making has applied judgment analysis to the decision making of professionals working in sectors including health and social care as well as banking; the common aim being to capture how different professional groups make certain key decisions in order to enhance the decision making of novice professionals. To date I have developed three web-based decision aids; the first two aids were funded for development by the Higher Education Academy and the ESRC respectively.

My first application of judgment analysis was in the field of community mental health as part of my MSc research, where I examined the factors influencing occupational therapists' decision making as to whether or not to accept a referral. This research began with a qualitative study, using interview and think aloud methodologies, with 8 experienced community mental health occupational therapists, to elicit the nature of information attended to when examining referral information. This research was extended as part of my $\mathrm{PhD}$; in collaboration with Professor Ken Gilhooly I conducted a quantitative study incorporating the key referral cues identified in the initial qualitative research phase in order to model 45 experienced occupational therapists' referral prioritisation policies. As well as comparing these objective policies with practitioners' subjective opinions of their policies, the objective policies were used to develop and test (using an RCT) a decision training aid for novice occupational 


\section{The Brunswik Society News/etter, 2013}

therapists, funded by the Higher Education Academy. The decision aid was found to have a positive effect on novices' decision making capacity.

I was then invited by Professor Mary Gilhooly (Principal Investigator) to be a cogrant holder on a New Dynamics of Ageing grant (ESRC administered), researching how different professional groups make decisions in cases of suspected elder financial abuse. In Phase I we interviewed professionals about actual incidents of suspected abuse to identify how they had detected and prevented abuse. In Phase II we statistically modelled decision making behavior across a large set of scenarios and measured consistency and discrimination in order to identify the experts amongst the sample. Phase III was a policy analysis of the relevant documents used by the three professional groups so this could be compared with practice behavior.

I subsequently applied for an ESRC-funded research grant with Co-grant holders Professor Mary Gilhooly and Professor Ken Gilhooly, to develop a web based training resource for health, social care and finance professionals based on the NDA research findings, which was tested for efficacy through a randomized controlled trial. This showed a statistically significant positive effect of the training on novices' capacity to detect and prevent elder financial abuse. This was then combined with a range of resources as part of a training website to enhance professionals' decision-making capacity in relation to elder financial abuse.

The website (www.elderfinancialabuse.co.uk) has had over 2000 visitors since it was launched in August 2012. The training resources produced have been endorsed for member use by Age UK, professional bodies such as the College of Occupational Therapists, CIFFAS Fraud detection Agency and the Building Society Association.

The practical applications of this research program in clinical domains has led to a number of approaches from researchers interested in applying the techniques of judgment analysis to decision making in their sectors of interest. I am currently collaborating with Professor Carolyn Unsworth (La Trobe University, Australia) on a United Kingdom Occupational Therapy Research Foundation funded project to explore how occupational therapy driving assessors determine whether an older or disabled patient is fit to drive. The research is at the stage of identifying expert judgment policy to develop training, and we will be undertaking a randomized controlled trial to determine the effectiveness of the training on student occupational therapists decision making next year.

I have also recently commenced a research project with Dr Mary Hickson (Imperial College London), funded by the Imperial College Healthcare Charity to explore how dieticians prioritize referrals for dietetic assessment in acute adult services. I am always interested in considering new areas of expert decision making research, so please contact me if you have any ideas for potential collaboration. 
References:

Davies, M. L., Gilhooly, M. L. M., Gilhooly, K. J., Harries, P. A., \& Cairns, D. (2013). Factors influencing decision-making by social care and health sector professionals in cases of elder financial abuse. European Journal of Aging. doi:10.1007/s10433-013-0279-3

Davies, M., Harries, P. A., Gilhooly, K. J., Gilhooly, M., Cairns, D., Notley, E., Penhale, B., Stanley, D., Gilbert, A., \& Henessey, C. (2011). Factors used in the detection of elder financial abuse: A judgment and decision making study of social workers and their managers. International Social Work, 54(3), 404-420. doi:10.1177/0020872810396256

Gilhooly, M. L. M., Cairns, D., Davies, M., Harries, P. A., Notley, E., \& Gilhooly, K. J. (2013). Decision cues, pathways to detection and barriers to action. Journal of Adult Protection. doi:10.1108/14668201311313578

Harries, P. A., Davies M., Gilhooly, K. J., Gilhooly, M. L. M., \& Cairns, D. (2013). Detection and prevention of financial abuse against elders. Journal of Financial Crime. Early online 20/12/2013. doi:10.1108/JFC-05-2013-0040

Harries, P., \& Gilhooly, K. (2003). Identifying occupational therapists referral priorities in community health. Occupational Therapy International, 10(2), 150-164. doi:10.1002/oti.182

Harries, P. A., \& Gilhooly, K. (2003). Generic and specialist occupational therapy casework in community mental health. British Journal of Occupational Therapy, 66(3), 101-109.

Harries, P., \& Gilhooly, K. (2011). Training occupational therapists to make expert, occupationally focused, community mental health referral decisions. British Journal of Occupational Therapy, 74(2) 58-65. doi:10.4276/030802211X12971689813963

Harries, P., Tomlinson, C., Notley E., Davies, M., \& Gilhooly, K. (2012). Effectiveness of a decisiontraining aid on referral prioritisation capacity: A randomized controlled trial. Medical Decision Making, 32(6), 779-791. doi:10.1177/0272989X12443418

Weiss, D., Shanteau, J., \& Harries, P. (2006). People who judge people. Journal of Behavioral Decision Making, 19, 441-454. doi:10.1002/bdm.529

\title{
A Contribution to the Debate on Coherence versus Correspondence: Examples of Incoherencies in Regulated Medical Markets
}

\author{
Professor Dr Christine Huttin \\ ENDEPUSresearch, Inc, USA \\ University Aix Marseille, FRA \\ Contact: chris.huttin@comcast.net
}

In 2009, the Brunswik society revisited the debate on the use of coherence versus correspondence theories especially in medicine (Dunwoody, 2009; Tape, 2009).

This contribution to the newsletter aims to provide several examples which illustrate the existence of incoherencies in regulated markets, due to bureaucratic processes and lack of adjustment with market mechanisms. It shows that the use of correspondence theories is particularly useful for the analysis of pharmaceutical markets, especially in highly regulated environments.

The first example concerns user fee policies in socialized health care systems, where exemption mechanisms and price regulation procedures can lead to incoherent pricing structures. For instance, some governmental policies set user fee levels at a much higher level than the production prices; this is considered as an incentive mechanism for switching from prescribed medicines to OTC medicines. However, the 


\section{The Brunswik Sogiety

clinical consequences of such shifts are uncertain and not usually assessed by national agencies in a national government. This may also be the result of different bargaining power between the two main healthcare professions involved - physicians and pharmacists - since switching Rx drugs to OTC drugs mainly benefits the pharmacist profession.

The second example is based on a comparison between branded medicines and generics, where generic prices are higher than originators and branded generics (generally from originators). This usually happens in countries where the drug price regulation is set per company on the basis of their product portfolio. In such a case, prices are the result of a negotiation between the government and the company who usually prefers to set higher prices for new drugs but accepts then to charge much lower prices for former originator drugs (e.g., even lower than their generic competitors). When this regulation based at a company level between the national price regulatory agency and a company prevails on a medical market, the price reduction at the producer level or distribution level is not usually passed to the consumer and the split is more directly shared between government and business. Therefore, the business-government negotiation results in high discrepancy of price structures for consumers and a lack of coherence, especially in the use of multiple therapies. In socialized systems, the share which remains with the government in a global budgeting system is then redistributed under the social contract in place, but not directly to the patients in need of the medications.

The third example concerns the lack of coherence between international clinical guidelines, especially for first line therapy, and physicians' treatment choices in different health systems for similar clinical cases but different budget constraints. Among barriers to adoption of guidelines, implicit restraints linked to economics of patients, products or physicians can lead groups of physicians, sensitive to similar types of cost cognitive cues, to different treatment choices (Huttin, 2004). Characteristics of the financing systems that may differently influence physicians in different systems are for instance: differences in accountability of their profession for the management of drug budgets and referral processes to secondary care; different relative prices for drug classes in different states or health care organizations, due to different price control regulations in socialized markets; or different discounted practices in more competitive markets.

In a context of a global agenda for health, a research agenda may be needed to understand the impact of such incoherencies, especially when international guidelines are adopted in market structures with different rates of diffusion of evidence and rates of adoption of new drugs.

A Reversed Conjoint Analysis of Physicians' Cost Sensitivity can Enhance the Role of State Preference Studies for Calibration of Econometric Models of Health Care Expenditures

The use of judgment research helps to explore critical decision points in clinical practice; it also helps to interpret trends in health care expenditures that remain unexplained with administrative surveys. Therefore, it is relevant to discuss some statistical methodological issues for this type of studies, and the special application called reversed conjoint studies (see Huttin, 2004): the embedding/scope issue and the existence of hypothetical biases. They were recently discussed by Professor 
Hausman and Professor Carson for the contingent valuation approach in willingness to pay studies. A reversed conjoint analysis of physicians' cost sensitivity aims to explain implicit restraints due to financial or economic information interfering with decision choices in clinical practice. Such an application shows the value of judgment research and such stated preference studies; especially with this topic of patient economics, it helps not to limit its measure to out of pocket costs or copayments.

The Brunswik society could possibly contribute to this topic with discussion of efficiency and relevant development of statistical tests in combination with efficiency tests used in econometric models run with effective data. The lens model is still useful to understand some human decisions especially with the increase of genomic medicine and lack of, or poor, evidence for subpopulations. Such studies can help to adjust predictors in revealed preference analysis.

\section{References:}

Dunwoody, P. T. (2009). Introduction to the special issue: coherence and correspondence in judgment decision making. Judgment and Decision Making, 4,113-115.

Huttin, C., \& endepresearch group. (2003). Patient charges and decision making behaviours of consumers and physicians. los Press ( www.iospress.nl ).

Huttin, C. (2012). New type of price measurement for medical services: interest of a cost sensitivity index for a research agenda on pharmaceutical models. Technology and Health Care, 20, 463-476.

(See also: "New cost sensitivity measures to assess impact of reimbursement systems on physicians "treatment choices based on an adaptation of the Lens model on cost related cues". Presentation at the Brunswik Society meeting, Minneapolis, 2004).

Tape, T. G. (2009). Coherence and correspondence in medicine. Judgment and Decision Making, 4, 134-140.

\section{Mobile Technologies and the Promise of Representative Design and Representative Sampling ${ }^{2}$}

\author{
Robert M. Kaplan \\ Office of Behavioral and Social Sciences Research, \\ National Institutes of Health,
}

US

\section{Contact: robert.kaplan@nih.gov}

It is an honor to receive an invitation to contribute to the newsletter of the Brunswik Society. In the 1930s, my father was a graduate student at Berkeley and Brunswik was a member of his doctoral exam committee. This had a profound impact on the way my father thought about science and, perhaps, what values were transmitted to me. Even in the 1930s, Brunswik recognized that laboratory experiments that manipulated one variable at a time were likely to lead to nongeneralizable results. If we have learned anything in the last 70 years, it is that context matters.

\footnotetext{
${ }^{2}$ The note was adapted from: Kaplan, R. M. \& Stone, A. A. (2013).
} 


\section{The Brunswik Society

Nonrepresentative Environments

Science and the practice of health care typically involve bringing patients to the controlled environment of a laboratory or clinic. Despite the advantages of the psychological laboratory, Brunswik was among the first to emphasize that most outcomes of interest are determined by or modified by a wide variety of contextual factors. What happens in the laboratory may not be representative of what happens in more complex non laboratory environments. These issues have not changed since Brunswik's time. But what has changed is the capability to study behavior in a wide range of natural environments and to understand the effects of context. We now have methods that sample experiences from every day environments and circumstances. New technologies, including cell phones, sensors, and monitors now make it possible to collect information outside of the laboratory in environments representative of everyday life. In collaboration with Arthur Stone from Stony Brook University, I recently reviewed the role of mobile technologies in the assessment of health related behaviors, physiological responses, and self reports (Kaplan \& Stone, 2013).

\section{Mobile Technologies.}

An important advance in bringing the laboratory and clinic into natural environments is the rapid development of new portable communications technologies. Ecological momentary assessment (EMA) involves sampling current behaviors and experiences as they occur in the natural environments of everyday life (Shiffman, Stone, \& Hufford, 2008). This revolution in technology enables studies with representative designs to provide the scientific underpinnings of what Brunswik advocated (Brunswik, 1955). On the clinical side, ecological momentary interventions (EMI) are a direct outgrowth of EMA. EMI methods are intended to take interventions (usually behavioral) into the everyday lives of patients. These interventions are administered via mobile devices and are momentary in that they happen immediately in the natural environment (hence the analogy with EMA).

The International Telecommunications Union estimated that about 5.6 billion cell phones are now in use around the world and that this number will at least double (far exceeding the number of people in the world) within the next decade. China, for example, has nearly one billion wireless accounts, and the United Arab Emirates has nearly two active wireless accounts for each person in the population. Wireless communication devices have leapfrogged wired systems. About $91 \%$ of US adults use a mobile phone regularly. Financial resources have not hindered widespread dissemination of these technologies: The fast-growing markets include African American and Hispanic users and low-income families. Growing evidence indicates that electronic technologies may be the best way to help low-income people change behavior (Bennett et al., 2012). The almost universal availability of cell phones means that most people are connected to a data collection and intervention apparatus, allowing the implementation of representative designs. Technologies are also available to collect real-time psycho-physiological responses. Mobile technologies can be used to capture, store, analyze, manage, and present population-level data, including geographic information system (GIS) data, and potentially to deliver these data more efficiently. Research using these technologies could lead to the development of early detection and warning systems to address possible disease outbreaks. Further, these tools are now being used in clinical trials to capture outcomes in natural environments and reduce burden on participants. Remote monitoring and sensing can allow 


\section{The Brunswik Society

researchers to recruit and follow patients without the need and associated costs of transporting them to a research or health care setting. As a result, the trials may get higher-quality data at lower costs.

\section{Do Mobile Health Interventions Work?}

Despite the promise of mHealth technologies, we still have relatively little evidence that they can be used to change behavior. Stone and I reviewed the 21 trials that we were able to locate in the published literature (Kaplan \& Stone, 2013). One trial used mHealth intervention in all conditions and only varied content. Among the remaining 20 trials, $11(55 \%)$ failed to document a clear benefit of mHealth interventions and another $3(15 \%)$ had mixed results. About half of the trials on smoking cessation achieved a benefit of text-message-based intervention, whereas the record was less clear for weight loss. Most trials failed to show that text messaging improved diabetes outcomes. To date, the most consistent positive results are for improving adherence to antiretroviral therapy among adults with HIV in the developing world. Overall, most of the trials we evaluated failed to show a significant benefit of the mHealth interventions and only 6 of 20 trials (30\%) demonstrated a clear and unambiguous benefit across multiple measures as assessment periods. The availability of the new mobile laboratory is likely to have a profound effect on clinical practice and on research. But, we need more persuasive evidence that they result in beneficial behavior change.

Our review suggests that mobile electronic technologies have already had a profound effect on measurement. Developments in ambulatory assessment and ecological momentary assessment have ushered in important advances in our ability to study behavior outside of the laboratory. They have made the notion of representative design and representative sampling a practical reality.

In contrast to our positive assessment of the role of new wireless technologies for assessment, we remain open-minded yet skeptical about the value of mobile technologies for changing health behavior and for improving the health of human populations. Although there is clearly great promise, most systematic trials fail to support the value of mHealth interventions. Additional trials are needed, and we most strongly encourage evaluations of applications that are based on evidence-based principles of behavior change.

\section{Realizing Brunswik's Contribution}

Brunswik suggested that acquiring large samples of individuals was less important than collecting observations of a few individuals in large representative samples of situations (Brunswik, 1955). Progress in understanding the impact of behavioral settings has been slow because it is very difficult to observe individuals in the wide range of circumstances that they encounter. Such research is extremely demanding, expensive, and rarely feasible. However, new technologies and methodologies now enable the collection of data in a wide range of natural environments. With informed consent, we are now capable of capturing information in the natural physical and social environment. We are hopeful that these new technologies will help us make feasible the science that Brunswik demanded. 
References:

Bennett, G. G., Warner, E. T., Glasgow, R. E., Askew, S., Goldman, J., Ritzwoller, D. P., . . . Be Fit, Be Well Study Investigators. (2012). Obesity treatment for socioeconomically disadvantaged patients in primary care practice. Archives of Internal Medicine, 172(7), 565-574. doi: 10.1001/archinternmed.2012.1

Brunswik, E. (1955). Representative design and probabilistic theory in a functional psychology. Psychological Review, 62(3), 193-217.

Kaplan, R. M., \& Stone, A. A. (2013). Bringing the laboratory and clinic to the community: Mobile technologies for health promotion and disease prevention. Annual Review of Psychology, 64, 471498. doi:10.1146/annurev-psych-113011-143736

Shiffman, S., Stone, A. A., \& Hufford, M. R. (2008). Ecological momentary assessment. Annual Review of Clinical Psychology, 4, 1-32.

\title{
Interested in Brunswikian Literature?
}

\author{
Esther Kaufmann \\ University of Zurich \& University of Konstanz \\ $\mathrm{CH}, \mathrm{GER}$
}

Last summer Gunhild Wolf gave me the library by Bernhard Wolf which is listed below. The goal of this list is to make the scientific community aware of Brunswik's heritage - and also the link to Heider. Please let me know if you have any ideas how we can make this literature available for interested researchers. Thank you - also to Gunhild Wolf for her gift on behalf of Bernhard to interested researchers worldwide. Please let me know if you need a copy of the articles marked by $a^{*}$ esther.kaufmann@gmx.ch.

\section{Charlotte Bühler}

Bühler, C. (1929). Das Seelenleben des Jugendlichen: Versuch einer Analyse und Theorie der psychischen Pubertät (5. Aufl.). Verlag von Gustav Fischer: Jena.

Bühler, C. (1928). Kindheit und Jugend: Genese des Bewusstseins. Verlag von S. Hirzel in Leipzig.

\section{Egon Brunswik}

*Brunswik, E. (1927). Strukturmonismus und Physik. Unpublished Dissertation, Philosophische Fakultät der Universität, Wien. (Copy: I)

Brunswik, E. (1928). Zur Entwicklung der Albedowahrnehmung. Zeitschrift für Psychologie, 109, 40-115. (Copy: I)

Brunswik, E. (1929). Prinzipienfragen der Gestalttheorie. In E. Brunswik, C. Bühler, H. Hetzer, L. Kardos, E. Köhler, J. Krug, \& A. Willwoll (Eds.), Beiträge zur Problemgeschichte der Psychologie: Festschrift zu Karl Bühlers 50. Geburtstag (pp. 78-149). Jena: G. Fischer. (Hard Copy: I, Copy: I)

*Brunswik, E. (1930). Über Farben-, Grössen- und Gestaltkonstanz in der Jugend. In H. Volkelt (Ed.), Bericht über den 11. Kongress für experimentelle Psychologie in Wien 1929 (pp. 52-56). Jena: G. Fisher.

*Brunswik, E., Goldscheider, L., \& Pilek, E. (1932). Untersuchungen zur Entwicklung des Gedächtnisses bei Knaben und Mädchen vom 6-18 Jahren. Zeitschrift für angewandte Psychologie, Beiheft 64, VIII+158. (Hard Copy: I, Copy: I)

*Brunswik, E. (1933). Die Zugänglichkeit von Gegenständen für die Wahrnehmung und deren quantitative Bestimmung. Archiv für die gesamte Psychologie, 88, 377-418. (Copy: I)

*Brunswik, E. (1934). Flächeninhalt und Volumen als Gegenstände der Wahrnehmung. In O. Klemm (Ed.), Bericht über den 13. Kongreß der Deutschen Gesellschaft für Psychologie in Leipzig (pp. 120-123). Jena: G. Fischer. (Copy: I)

*Brunswik, E. (1934). Wahrnehmung und Gegenstandswelt: Grundlegung einer Psychologie vom Gegenstand her. Leipzig und Wien: F. Deuticke (Habilitationsschrift). (Hard Copy: I, I) 
Brunswik, E. (1935). Experimentelle Psychologie in Demonstrationen. Wien: J. Springer. (Hard Copy: I, I, I, Copy: I)

*Brunswik, E. (1946). Points of view (pp. 523-537). In P. L. Harriman (Ed.), Encyclopedia of Psychology. New York: Philosophical Library, Inc. (Hard copy: I)

Brunswik, E. (1947). Systematic and representative design of psychological experiments. With results in physical and social perception. Berkeley: University of California Press. (Copy: I)

Brunswik, E. (1952). The Conceptual Framework of Psychology, International Encyclopedia of Unified Science (Vol. 1, No. 10, pp. IV + 102). Chicago: University of Chicago Press (Hard Copy: 7 exemplare)

Brunswik, E. (1956). Perception and the representative design of psychological experiments. Berkeley: University of California Press. (Hard copy: I, I, I)

*Brunswik, E. (1957). Scope and aspects of the cognitive problem. In H. Gruber, K. R. Hammond, \& R. Jessor (Eds.), Contemporary approaches to cognition (pp. 5-31). Cambridge: Harvard University Press. (Hard copy: I, I)

*Brunswik, E. (1966). Reasoning as a universal behavior model and a functional differentiation between "perception" and "thinking". In K. R. Hammond (Ed.), The Psychology of Egon Brunswik (pp. 487494). New York: Holt, Rinehart and Winston. (Hard Copy: I, I, I)

\section{Fritz Heider}

*Heider, F. (1920). Zur Subjektivität der Sinnesqualitäten (On the subjectivity of sense qualities). Unpublished dissertation. University of Graz.

Fritz Heider: The Notebooks". Vol. 1: Methods, Principles and Philosophy of Science. Heider, F. Benesh-Weiner, Marijana (Ed.). Weinheim Psychologie Verlags Union (1987).

Fritz Heider: The Notebooks“. Vol. 2: Perception. Heider, F. Benesh-Weiner, Marijana (Ed.). Weinheim Psychologie Verlags Union (1988).

Fritz Heider: The Notebooks". Vol. 3: Motivation. Heider, F. Benesh-Weiner, Marijana (Ed.). Weinheim Psychologie Verlags Union (1988).

Fritz Heider: The Notebooks“. Vol. 4: Balance Theory. Heider, F. Benesh-Weiner, Marijana (Ed.). Weinheim Psychologie Verlags Union (1988).

Fritz Heider: The Notebooks". Vol. 5: Attributional and interpersonal evaluation. Heider, F. BeneshWeiner, Marijana (Ed.). Weinheim Psychologie Verlags Union (1988).

Fritz Heider: The Notebooks“. Vol. 6: Units and coinciding Units. Heider, F. Benesh-Weiner, Marijana (Ed.). Weinheim Psychologie Verlags Union (1989).

\section{Ernst Meumann}

Meumann, E. (1922). Vorlesung zur Einführung in die experimentelle Pädagogik und ihre psychologischen Grundlagen: 1. Band. Verlag von Wilhelm Engelmann: Leipzig. (Hard copy: I)

Meumann, E. (1922). Vorlesung zur Einführung in die experimentelle Pädagogik und ihre psychologischen Grundlagen: 2. Band. Verlag von Wilhelm Engelmann: Leipzig. (Hard copy: I)

Meumann, E. (1922). Vorlesung zur Einführung in die experimentelle Pädagogik und ihre psychologischen Grundlagen: 3. Band. Verlag von Wilhelm Engelmann: Leipzig. (Hard copy: I)

\section{Bernhard Wolf}

Wolf, B. (1995). Brunswik und ökologische Perspektiven in der Psychologie. Deutscher Studien Verlag: Weinheim.

Wolf, B. (2008). Principles of Brunswik's Probabilistic Functionalism. Landau: Verlag Empirische Pädagogik.

\section{Additional}

Bierbrauer, G. (1983). Kurt Lewins Leben, Werk und Wirkungsgeschichte in der Emigration. Psychologische Forschungsberichte aus dem Fachbereich 8 der Universität Osnabrück.

Hammond, K. R., \& Wascoe, N. E. (1980). New directions for methodology of social and behavioral science: Realizations of Brunswik's Representative Design. Jossey- Bass Inc.,: San Francisco.

Fischer, K. R., \& Stadler, F. (1997). Wahrnehmung und Gegenstandswelt: Zum Lebenswerk von Egon Brunswik. Springer: Wien, New York. 


\title{
The Brunswik Society News/etter, 2013
}

\section{A Critical Meta-Analysis of Lens Model Studies in Human Judgment and Decision-Making}

\author{
Esther Kaufmann \\ University of Zurich \& University of Konstanz, \\ $\mathrm{CH} / \mathrm{GER}$ \\ Ulf-Dietrich Reips \\ University of Konstanz, \\ GER \\ Werner W. Wittmann \\ University of Mannheim, \\ GER
}

Contact: esther.kaufmann@gmx.ch

A part of our analysis on judgmental achievement has been accepted for publication by PLoS One (Kaufmann, Reips, \& Wittmann, in press). In this study we applied a Brunswikian based psychometric meta-analysis (see Wittmann, 1985, 2009) to lens model studies on judgmental achievement. In this current study we conduct the first psychometric meta-analysis of judgmental achievement studies, in which we correct for a number of study design artifacts (e.g., measurement error, dichotomization), which may have potentially biased estimations (e.g., of the variability between studies) and led to erroneous interpretations (e.g., with regard to moderator variables). We identified 31 lens model studies $(\mathrm{N}=1151, \mathrm{k}=49)$ that met our inclusion criteria. We evaluated overall judgmental achievement as well as whether judgmental achievement depended on decision domain (e.g., medicine, education) and/or the level of expertise (expert vs. novice). We also evaluated whether using corrected estimates affected conclusions with regard to the success of bootstrapping with psychometrically-corrected models. Comparison of the results of the psychometric meta-analysis with the results of a traditional meta-analysis (which only corrected for sampling error) indicated that artifact correction leads to a) an increase in values of the lens model components, b) reduced heterogeneity between studies, and c) more successful bootstrapping. We argue that psychometric meta-analysis is useful for accurately evaluating human judgment, and show the success of bootstrapping.

Due to this study we are focusing now on further analysis of bootstrapping models in detail. As in our previous analysis, we start the analysis at the individual level and would like to compare it with studies at the aggregated - across individuals level. However, as this analysis is in preparation (Kaufmann \& Wittmann, 2013), we greatly appreciate any comments and ideas for our work, and would like to thank you for any interest in it.

\section{References:}

Kaufmann, E., Reips, U.-D., \& Wittmann, W. W. (accepted). A critical meta-analysis of lens model studies in human judgment and decision-making. PLOS ONE.

Kaufmann, E., \& Wittmann, W. W. (2013). The success of bootstrapping models under the lens. Working paper. University of Zurich \& University of Mannheim. 


\title{
The Brunswik Soeilety

Wittmann, W. W. (1985). Evaluationsforschung: Aufgaben, Probleme und Anwendungen. [Evaluation research: Tasks, problems and applications]. Berlin, Germany: Springer- Verlag.

Wittmann, W. W. (2009). Evaluationsmodelle. In H. Holling (Ed.). Enzyklopädie der Psychologie. Themenbereich B Methodologie und Methoden. Serie IV Evaluation - Band 1. Grundlagen und statistische Methoden der Evaluationsforschung (pp. 59-98). Göttingen: Hogrefe.

\section{News from the Past Year}

\author{
Jeryl L. Mumpower \\ U. S. National Science Foundation, \\ on leave from Texas A\&M University, \\ US
}

\section{Contact: jmumpowe@nsf.com}

During the past year, I have been on leave from my position at Texas A\&M University and have served as Division Director for Social and Economic Sciences at the U.S. National Science Foundation. I am beginning the second year in this role. This has been an interesting experience and I believe I am engaged in important, valuable work, but it has certainly affected my ability to get research done. Nonetheless, I have had some modest success with respect to publications during the past year.

My colleagues at Texas A\&M University, Arnie Vedlitz, Liu Shi, Jim Stoutenborough and I just published a paper in Risk Analysis entitled "Psychometric and Demographic Predictors of the Perceived Risk of Terrorist Threats and Willingness-to-Pay for Risk Management Programs." I also published a paper entitled "Low-Level Radioactive Waste Disposal Facility Siting: Lessons from the Past, Recent Developments, Future Prospects" in the International Journal of Policy Studies. Gary McClelland at the University of Colorado, Boulder, and I presented a paper entitled "A Signal Detection Analysis of Racial and Ethnic Disproportionality in the Referral and Substantiation Components of the U.S. Child Welfare Services System" at the SPUDM conference in Barcelona in August. Gary and I are now making revisions to the paper for publication in Judgment and Decision Making. Finally, Tom Stewart, Jim Holzworth and I continue to work on our next paper stemming from our research program investigating how people make selection and detection decisions (e.g., how they to decide whether to hire someone or whether a patient has a disease) in the face of uncertainty and different feedback conditions.

\section{References:}

Mumpower, J. L., Shi, L., Stoutenborough, J. W., \& Vedlitz, A. (2013). Psychometric and demographic predictors of the perceived risk of terrorist threats and the willingness to pay for terrorism risk management programs. Risk Analysis, 33(10), 1802-1811.

Mumpower, J. L. (2013). Low-level radioactive waste disposal facility siting: Lessons from the past, recent developments, future prospects. International Journal of Policy Studies, 4(1), 27-61. 


\title{
The Brunswik Society News/etter, 2013
}

\section{Cue Polarity Effects in Learning: A “Matching Bias" for Multiple Cue Judgment}

\author{
Jonathan J. Rolison \\ School of Psychology, Queen's University Belfast, \\ UK
}

Contact:j.rolison@qub.ac.uk

Cue polarity in multiple cue judgment refers to the direction of association between cues and a criterion. For cues that have a positive polarity, high cue values are associated with high criterion values, such as when high blood pressure (i.e., hypertension) predicts increased risk of heart disease and stroke. In contrast, cue and criterion values are inversely related for cues that predict negatively, such as when low blood pressure (i.e., hypotension) is associated with symptoms of dizziness and blurred vision.

My colleagues and I investigated how people learn positive and negative cuecriterion associations across a number of studies using the multiple cue probability learning paradigm (Rolison, Evans, Dennis, \& Walsh, 2012; Rolison, Evans, Walsh, \& Dennis, 2011). Our studies revealed that people are better able to learn positive than negative associations when relevant cues are combined with irrelevant cues in a multiple cue learning environment. We found that negative cue learning, but not positive cue learning, correlated with individual differences in working memory capacity (WMC) and participants' explicit beliefs about the relevance of the cues (Rolison et al., 2011, 2012). However, negative cue learning no longer correlated with participants' WMC or their explicit beliefs when a multiple cue task was preceded by training on a task that contained only positive relevant cues (Rolison et al., 2011). We concluded that positive cues are processed automatically by implicit associative processes that are independent of controlled attention and explicit reasoning.

More generally, there appears to be something quite automatic about processing compatible information such as with positive cue learning. Compatibility effects are well documented in studies of perceptual processing. When responding to the location of a stimulus on a computer monitor, people are faster to respond when stimulus and response sets are compatible, such as when a stimulus that appears to the left corresponds to a left key press (Eimer, 1995). Similar to our studies of multiple cue judgment, stimulus-response compatibility appears to prime the response that corresponds with the stimulus, suggesting that the brain may be hard-wired to process compatible mappings (Kornblum, Hasbroucq, \& Osman, 1990). In our studies of multiple cue judgment we found that irrelevant cues often attracted a slight positive weighting in participants' judgments, despite being irrelevant to predicting the criterion (Rolison et al., 2011, 2012).

Cue polarity effects in multiple cue judgment are strikingly similar to matching bias effects observed in studies of human reasoning (Evans, 1998). In the Wason selection task, participants are asked to select among a set of instances (i.e., labeled 


\title{
The Brunswik Sogiety

cards that can be turned over) those that would falsify a proposition (i.e., a rule that determines what labels are expected on either side of the cards). People show a strong tendency to select cards with labels that match the card labels mentioned in the rule, rather than select the cards that would falsify the rule. In dual-process theories of reasoning it is proposed that this matching bias is generated by an implicit heuristic that must be inhibited by analytical thinking for one to reason logically about the task (Evans, 2003).

Our studies of cue polarity effects in multiple cue judgment suggest that there is something special about positively predictive cues. In contrast with negatively predictive cues, positive cues are learned effortlessly and automatically even in complex multiple cue environments. Our findings parallel similar observations in a variety of domains, from low-level perceptual processing to high-level reasoning. These commonalities across research domains raise the intriguing possibility that similar psychological mechanisms might be involved.

\section{References:}

Rolison, J. J., Evans, J. St. B. T., Dennis, I., \& Walsh, C. R. (2012). Dual-processes in learning and judgment: Evidence from the multiple cue probability learning paradigm. Organizational Behavior and Human Decision Processes, 118, 189-202.

Rolison, J. J., Evans, J. St. B. T., Walsh, C. R., \& Dennis, I. (2011). The role of working memory capacity in multiple-cue probability learning. Quarterly Journal of Experimental Psychology, 64, 1494-1514.

\section{Improving Referees' Decisions in Sports}

\author{
Geoffrey Schweizer \& Henning Plessner \\ University of Heidelberg, \\ GER
}

\author{
Ralf Brand \\ University of Potsdam, \\ GER
}

Contact: geoffrey.schweizer@issw.uni-heidelberg.de

Referees' decisions play an important role in sports like soccer and basketball. In order to ensure a fair competition, referees' decisions are supposed to be as accurate as possible. Among other decisions, referees have to discriminate between foul and legal play and they have to judge the severity of foul play. As a substantial proportion of referees' decisions is wrong, training methods for referees' decisions are needed. However, progress in this field used to be slow as theoretical understanding of referees' decision making was limited.

We suggest that understanding referees' decisions can be improved by using the lens model framework (Plessner, Schweizer, Brand, \& O'Hare, 2009). Referees have to judge a distal criterion (e.g., foul play or legal play) by taking multiple cues (e.g., players' positions, players' speed, position of the ball) into account. The more closely referees' cue utilization mirrors ecological validities, the more accurate are their 


\section{The Brunswik Society News/etterer, 2013}

decisions. As correlations between cues and criteria are probabilistic, there will always be a proportion of incidents where referees cannot make correct decisions. From an applied perspective, it is important that their decisions are nevertheless predictable.

Based on these considerations we developed a video-based training method. Referees have to watch videos, make decisions and get feedback on the correctness of their decisions. Several experimental studies using control groups as well as different training groups indicate that the training method is indeed able to improve referees' decisions. First, referees who participate in the training program improve their ability to discriminate between options (e.g., foul play or legal play) and to judge the severity of foul play (Schweizer, Plessner, Kahlert, \& Brand, 2011). Second, video training can influence referees' response tendency, leading to predictable decisions in ambiguous situations (Schweizer, Plessner, \& Brand, 2013). Interestingly, results are in line with the assumption that referees' decisions are mainly based on automatic processing: Outcome feedback without further explanations is sufficient for learning success. Only immediate feedback leads to learning effects, whereas delayed feedback does not.

Understanding referees' decisions using the lens model framework has proven beneficial for developing training methods. The lens model might prove even more useful for future work: Constructing a lens for referees' decisions in certain situations might help to understand why referees make certain mistakes and how to support them avoiding these mistakes in the future. Our work so far suggests that the lens model and social judgment theory are not only valuable for primarily theoretical research, but that, quite the contrary, they can be exceptionally helpful for getting access to applied problems in judgment and decision making.

\section{References:}

Plessner, H., Schweizer, G., Brand, R., \& O'Hare, D. (2009). A multiple-cue learning approach as the basis for understanding and improving soccer referees' decision making. In M. Raab, J. Johnson \& $\mathrm{H}$. Heekeren (Eds.), Progress in brain research: Mind and motion: The bidirectional link between thought and action (pp. 151-158). Amsterdam: Elsevier Press.

Schweizer, G., Plessner, H., \& Brand, R. (2013). Establishing standards for basketball elite referees' decisions. Journal of Applied Sport Psychology, 25, 370-375. doi:10.1080/10413200.2012.741090

Schweizer, G., Plessner, H., Kahlert, D., \& Brand, R. (2011). A video-based training method for improving soccer referees' intuitive decision-making skills. Journal of Applied Sport Psychology, 23, 429-442. doi:10.1080/10413200.2011.555346 


\title{
The Brunswik Society News/etter, 2013
}

\section{On the Relative Importance of Linear Model and Human Judge(s) in Combined Forecasting}

\author{
Matthias Seifert \\ Operations \& Technology Area, IE Business School, \\ ESP
}

Allègre L. Hadida

University of Cambridge Judge Business School and Magdalene College, UK

Contact: matthias.seifert@ie.edu / a.hadida@jbs.cam.ac.uk

The debate of when and to what extent managerial forecasts should rely on linear models or judgmental predictions lies at the heart of our study. Existing research discusses the strengths and weaknesses of using linear models or human judgments on their own (Dawes, Faust, \& Meehl, 1989; Lawrence, Goodwin, O'Connor, \& Önkal, 2006; Meehl, 1996). However, demonstrations of how a combination of both model and judge can lead to higher forecasting accuracy are still few and far between (Blattberg \& Hoch, 1990; Lawrence, Edmundson, \& O'Connor, 1986). The finding that a simple $50 / 50$ weighting between model outputs and managerial judgment is likely to outperform either of the two alone (Blattberg \& Hoch, 1990) had a profound impact on our understanding of the use of decision support tools and spawned several follow-up studies (for example, Hoch \& Schkade, 1996; Sanders \& Ritzman, 1995; Stewart, Roebber, \& Bosart, 1997). Our paper examines the robustness of the proposed 50/50 split in greater depth. In particular, we use empirical data from the music industry to analyze the influence of task structure, domain-specific expertise and judgment aggregations on the effectiveness of combined model-judge(s) forecasts.

\section{Empirical Context \& Data Collection}

The forecasting event we investigate is the entry position of pop music singles in national Top 100 charts. Before a single enters the music charts, it is preceded by a promotion period of eight to ten weeks during which it may be broadcast on radio and television and may appear in print and online media or as part of a promotional retail campaign. Forecasting the success of new artists entails a substantial amount of uncertainty about market performance and may therefore be described as an illstructured task. Conversely, the success of artists with an established, historical track record may be easier to anticipate, and its prediction may be described as a wellstructured task.

In order to identify the predictor variables to be included in our ecological model, we conducted 23 in-depth semi-structured interviews with senior record companies' Artists and Repertoire (A\&R) managers in the two largest music markets in Europe: Germany and the UK. We generated 210 cue profiles of as-yet unreleased pop singles from criteria identified through the interviews, and used online questionnaires to collect prediction data over a period of 12 weeks. Our sample comprises 92 A\&E managers 


\section{The Brunswik Society News/etter, 2013}

(the expert forecasters) and a control group of 88 graduate students in the UK and Germany (the novices).

\section{Methods}

Our research design is based on a Brunswikian lens model as used in judgment analysis (Brunswik, 1956; Cooksey, 1996). We follow a regression procedure introduced by Blattberg and Hoch (1990) to assess the predictive accuracy of a linear, best fitting model of the task ecology combined with single as well as aggregated judgments. We also test whether task structure and domain-specific expertise moderate the optimal relative importance of linear model outputs and individual judgment by performing a 2x2 factorial ANOVA using "task structure" and "domainspecific expertise" as independent variables and the "optimal relative split between model and judge" (a factor derived from the lens model analysis) as the dependent variable. Our key interest lies in understanding human judges' ability to extract predictive value from contextual information associated with the forecasting problem, thereby improving the accuracy of linear models considered on their own (see also Blattberg \& Hoch, 1990; Hoch, 1987, 1988; Stewart et al., 1997).

\section{Results and Discussion}

Music experts generally achieve the highest predictive accuracy, pick up the highest percentage of unexplained model variance, and exhibit the greatest validity of expertise among all forecasters. As expected, linear models outperform the respondents least familiar with the predictive task at hand (novices), and individual expert judgment proves most powerful when experts assess the chart entry position of newcomers. When making predictions in ill-structured tasks conditions, experts pick up 8 times more unexplained model variance than novices. These individual-level effects are consistent across the number of respondents included in each model-multiple judge combination, regardless of the method used for aggregating these respondents' predictions. An increase in the number of aggregated judgments also decreases the relative importance of linear models, regardless of forecasting task structuredness and of the level of domain-specific expertise involved.

Our results extend Blattberg and Hoch (1990) by demonstrating that the proposed 50/50 split may be sensitive to changes in judges' levels of domain-specific expertise. Specifically, the results of our 2x2 factorial ANOVA indicates a positive relationship between the levels of domain-specific expertise and the relative importance of human judgment in combined forecasts. Our research also offers a reconciliation of contradictory findings relative to the predictive performance of linear models versus human judges (Lawrence et al., 2006; Makridakis et al., 1993), by supporting the view that their relative predictive performance may at least partly depend on the moderating effect of task structure.

Moreover, a significant interaction effect between domain expertise and task structure indicates that the model forecast takes precedence in well-structured tasks, regardless of domain-specific expertise. In contrast, when facing ill-structured forecasting tasks, an increase in domain-specific expertise shifts the optimal modeljudge split towards a heavier reliance on human judgment.

Finally, our study offers empirical insights into the value of model-multiple judge combinations associated with mechanically aggregated group judgments. When 
considering ill-structured forecasting tasks, the increased value of aggregated judgments appears related to an improvement in the collective ability to interpret nonlinearities in the task environment. Conversely, when considering well-structured tasks, superior forecasting performance is likely to result from a more efficient interpretation of linear relationships in the task ecology. These empirical findings therefore outline the conditions in which it is desirable to employ expert judges when generating predictions.

References:

Blattberg, R. C., \& Hoch, S. J. (1990). Database models and managerial intuition: $50 \%$ model $+50 \%$ manager. Management Science, 36(8), 887-899.

Brunswik, E. (1956). Perception and the representative design of psychological experiments ( $2^{\text {nd }}$ ed.). Berkeley, CA: The University of California Press.

Cooksey, R. (1996). Judgment analysis: Theory, methods and applications. NY: Academic Press.

Dawes, R., Faust, D., \& Meehl, P. E. (1989). Clinical versus actuarial judgment. Science, 243(4899), 6874.

Hoch, S. J. (1987). Perceived consensus and predictive accuracy: The pros and cons of projection, Journal of Personality and Social Psychology, 53(2), 221-34.

Hoch, S. J. (1988). Who do we know: Predicting the interests and opinions of the American consumer. Journal of Consumer Research, 15(3), 315-24.

Hoch, S. J., \& Schkade, D. A. (1996). A psychological approach to decision support systems. Management Science, 42(1), 51-64.

Lawrence, M., Edmundson, R., \& O'Connor, J. F. (1986). The accuracy of combining judgmental and statistical forecasts. Management Science, 32, 1521-1532.

Lawrence, M., Goodwin, P., O'Connor, M., \& Önkal, D. (2006). Judgmental forecasting: A review of progress over the last 25 years. International Journal of Forecasting, 22, 493-518.

Makridakis, S., Chatfield, C., Hibon, M., Lawrence, M., Mills, T., Ord, K., \& al., (1993). The M2 competition: A real time judgmentally based forecasting study. International Journal of Forecasting, 9, 5-22.

Meehl, P. E. (1996). Clinical versus statistical prediction: A theoretical analysis and a review of the evidence. Northvale, $\mathrm{NJ}$ : Jason Aronson.

Sanders, N., \& Ritzman, L. (1995). Bringing judgment into combination forecasts. Journal of Operations Management, 13, 311-321.

Seifert, M., \& Hadida, A. L. (2013). On the relative importance of linear model and human judge(s) in combined forecasting. Organizational Behavior and Human Decision Processes, 120, 24-36.

Stewart, T. R., Roebber, P. J., \& Bosart, L. F. (1997). The importance of the task in analyzing expert judgment. Organizational Behavior \& Human Decision Processes, 69(3), 205-219. 


\title{
The Brunswik Society Newsletter, 2013
}

\section{Path dependency and Routines: A Threat to Capability Development}

\author{
Lars Sjödahl \\ Lund University, Lund, \\ SWE
}

Contact: lars.e.sjodahl@gmail.com

Science begins with doubt.

Science stresses independence of judgment, not conformity.

Fang Lizhi: Axioms of Science, New York Review of Books, April 24, 2012

In economics research, Levinthal and Myat (1994), called attention to the fact that positive feedback or lack of negative feedback can lock future paths to current or new trajectories, like new theoretical or methodological approaches, development of new methods or skills (see also Arthur, 1994). In a similar way, Leonard-Barton (1992) emphasized the fact that unwillingness to abandon unproductive knowledge in favor of new knowledge (approaches) may turn existing behavior into core rigidity, hindering development of creativity and new capabilities. In her book "Creating Capabilities" Martha Nussbaum (2011, p. x, Preface) asks: "What are people actually able to do and to be? What real opportunities are available to them?"

In research, the amount of antecedent research publications and projects together with the feedback character (positive, negative or ignorance) can naturally result in path dependency. As bounded, rational decision makers, even researchers are susceptible to what is called path dependency, that is, not only influenced by outcomes and achievement but also by underlying factors like beliefs, attitudes, values and varying constraints. On an individual level, the single researcher may try to avoid path dependency by self-imposed discipline, a kind of bounded rationality. On a collective decision level this escape from path dependency is hardly possible. Any group that tries to decide by aggregating its members' views and preferences will run the risk of exchanging bounded rationality with a more or less rigid, bounded path dependency, that is, the collective decision is bound to be a compromise constrained by the decisions one has made in the past (Bennett \& Elman, 2006). It may be difficult to specify in concrete terms the forces and circumstances that promote resistance to path dependency. It might be easier, however, to list some factors likely to predispose for path dependency. This problem is discussed briefly by Linn Andersson (2013, pp. 22-24). It seems reasonable to assume that the following factors, in some way or another, are involved in the process leading to path dependency:

1. Complexity of the problem or tasks concerned

2. The environment's uncertainty, changeability with time

3. Interdisciplinary obstacles, eventual conflicting or competing ideas.

4. How does the feedback mechanism function, i.e. what are the signals from different consumers of the research findings? 


\section{The Brunswik Society

Path dependency may also be due to a tendency to frame problems and scenarios too narrowly (Kahneman \& Cavallo, 1993). In Brunswik (1956, p. 58) we find the following bold suggestion for how to avoid this narrowness by extending the sampling process as follows: "representative sampling is extended from the subjects to the objects, from the individuals to the stimulus situations and tests." Applying this definition in research practice means that we can refer our research findings to defined causal fields (von Wright, 1971) or to pre-defined ecological domains (Sjödahl, 2011, 2012)

Brunswik's suggestion to extend the sampling procedure to include also the object world (i.e., the subject's personal and impersonal ecology) has not been very successful (Gigerenzer, 2006, p. 250). This path dependency on classical sampling models hampers any deeper discussion about more complex causal relations (organism-environmental). Further, we would expect applicability of research findings to be less adequate if research designs exclude sampling of subjects' ecology.

Von Wright (1971), reminded us that the word "cause" is generally used with a multitude of meanings. In social sciences it is natural to expect that complex functional relations get different interpretations according to the interpreters' different political affiliations. This vagueness in interpreting our research results can be lessened if we select our experimental tasks as representing a wider, defined causal field, an ecological domain defined by a goal and expected consequences of possible decision alternatives. A meaningful task becomes a behavioral unit first when it represents an anterior phase and a posterior phase. The former represents a purpose, an intention, and the latter expected, imaged consequences of corresponding decision alternatives. Viewing behavior accordingly, many different research areas easily afford themselves for Brunswikian research, for example the following: 1) garden therapy, 2) visual and musical art perception, 3) social norms, 4) multisensory perception, 5) psychiatric diagnostics.

According to Simon (1947), decision makers seek to satisfy rather than maximize. This generalization certainly applies also to decision researchers choosing among presumptive research topics, theoretical and conceptual approaches. We would therefore expect that bounded rational researchers make different choices of research topics, methodological and theoretical approaches partly due to individual differences in aspiration level. It has been proposed by Cyert and March (1963) that decision makers evaluate alternatives according to their subjective availability and they make risk evaluations biased towards recent experiences (March, 1994). Bounded decision makers consider only a limited number of alternatives and a limited amount of information (ibid). Information regarding different alternatives is often perceived as ambiguous and complex and tends to result in a messy decision-process (Mintzberg, Raisinqhani \& Théorét, 1976).

One way out of such a situation may be to resort to routine behavior. Routines are described by Simon (1947) as behavior carried out automatically, triggered by a certain class of stimuli. A similar definition is found in Nelson and Winter (1982) who defined routines as regular and rigid behavior acts elicited by certain stimuli. Such classes of stimuli can very well consist of people, for example old people, psychotic people or children. It is a strange observation that complaints and shortcomings within the medical field are so often explained by referring to faulty routines, when just the routine character may be the culprit. You would expect that rigid behavior routines, 


\section{The Brunswik Society

quite useful sometimes, when failing would be remedied by case-learning and human judgment and not with a new routine. Maybe the diagnostic situation within some medical fields like psychiatry has become so hampered by practicalities like timerestraints, costs and failing competence-levels that what was formally regarded as qualified, professional skills has declined into routines. Two researchers, Kirk and Kutchins (1992) practicing in a psychiatric clinic, report the following observations: "The staff at one agency explained that diagnoses were the art of making distinction without differences. Psychiatrists would frequently debate the finer points about the correct diagnoses but would always prescribe Haldol regardless of the outcome of the debate." (p. 235)

It is a plausible assumption that psychiatric diagnostics as well as drug prescriptions, to a considerable extent, consist in routine behavior. When the Swedish National Board of Health and Welfare (1997) examined 8069 psychiatric drug-lists, the notes were found all through to be difficult to read and interpret. The report gives the following examples: "In 16 per cent of the cases information is missing regarding preparation form, strength of the drug, dose interval, date for starting or finishing medication. The doctor's signature is sometimes in the wrong place, sometimes it is altogether missing. Many patients are on many drugs, 36 per cent have more than 6 drugs simultaneously. The drug lists examined by the Swedish National Board of Health and Welfare had deficiencies implying that the patient's safety was neglected." (1997, pp. 61-62).

Amazingly, not a word is said about how this negligence may be related to the diagnostic notes and to follow up measures of the patient's eventual recovery process. When doctors' recording of simple, factual information is so routinely performed one cannot avoid asking: What about the quality of doctor's more complex, inferential, diagnostic notes? Are they also incomplete or routinely performed?

Further, the use of modern, prefabricated, non-etiological, symptom-lists like the DSM-manual can easily turn into routine use, that is, routine category-labeling of the patient and drug prescription. Allen Frances (2010), chairman of the task force for the descriptive psychiatric symptom manual (DSM-IV), reviewing his earlier work, expressed fears for a new, more inclusive DSM manual (DSM-V) with the following words; "DSM-V would create tens of millions of newly misidentified, false-positive patients. Thus greatly exacerbating the problems caused already by an overly inclusive DSM-IV. There would be massive overtreatment with medications that are unnecessary, expensive, and often quite harmful" (p. 1). Despite this warning the new manual, the DSM-V, has now been published.

Referring to the World Health Organization (WHO), Gigerenzer (2002) presented the following picture of the safety situation within medical care: "In the U. S. the average physician-patient contact is five minutes. Most of the information is presented in a vocabulary that is unintelligible to the patient. Patients tend to develop views of fate or "Inshalla" rather than learning to practice informed content. All is God's will, or the physician's, why should they worry? The Institute of Medicine estimated that some 44000 to 98000 patients are killed every year in U.S. hospitals by preventable medical errors and misadventures. It's as if one lived in a culture where death is a desirable transition from one life to a better one" (pp. 16-17). Comparing the medical field with commercial aviation Gigerenzer (ibid.) pointed out a reasonable cause for the poor safety situation within medical care: "But safety systems such as in 


\section{The Brunswik Society

commercial aviation... have not been set up in hospitals. In aviation, safety is an immediate interest of the pilot: if the passengers die in a crash the pilot will very likely die too. The situation vis-à-vis the doctor is different" (p. 21). It is quite possible that the degree of ego-involvement is reflected in different safety systems, just as Gigerenzer suggested. However, there are other differences between commercial aviation and the medical field related to their safety systems.

In commercial aviation the safety system is built on Flanagan's (1954) critical incident method, defined in terms of the activity goals, consequences of the action, and what is expected to be accomplished. Information of this sort becomes an excellent background for immediate feedback information, making learning possible in order to increase the safety level. Is there any similar safety mechanism aimed at learning by immediate task feedback within the medical field? It may be difficult to answer that question in general. However, let us take a brief look at the corresponding medical safety system in Sweden. For many years a special agency, the Medical Responsibility Board, has been in charge of complaints from patients and mandatory reports from hospitals on erroneous decisions and treatments. This arrangement was complemented in 2011 with a law saying that iatrogenic patient damage, that is, erroneous decisions and treatments, should be reported directly to the National Board of Health and Welfare. In cases of corrective measures the former organization (the Medical Responsibility Board) should still function, that is, suggest corrective measures. The bureaucratic arrangement was thus enlarged; did this enlargement help?

Let us look at some results from a study by Örstadius (2012). During 2011 only half the number of cases, usually dispatched during a year, was referred for correction. Besides thousands of cases dated 2011 there were still about 1400 complaints left over from 2010 waiting to be dealt with. This delay meant that many clients had to wait more than two years before receiving a response to their complaints. A consequence of this waiting is a delayed and limited task feedback to medical personnel and educational authorities. Neither learning nor motivational prerequisites for improving "on the spot" seems to be considered at all. Often, the patients' complaints are dispatched with a standard phrase like "the clinic has to revise its routines" or "the shortcoming depends on system errors". Neither routines nor system errors, however, are easy to correct without any learning opportunities. Sorry to say, this inertia or path dependency on routines seems to apply to the Swedish Medical Responsibility Board.

Quite a number of researchers, mainly within the commercial-economic field, have focused on the question: What triggers changes or breaks in routines or path dependency? This is an important question of relevance within many subject fields, obviously of the utmost concern within psychiatric diagnostics and drug prescription. It is, indeed, difficult to expect that any practice-based learning would elicit much change of routine behavior or path dependency in psychiatric diagnostics when you learn from the study by Groenier, Pieters, Halshof et al. (2008) that causal analyses were the least necessary elements in the diagnostic process according to 175 clinical psychologists.

As expected, researchers present different opinions about what triggers changes or breaks in ridged, routine behavior or path dependency. Gersick and Hackman (1990) suggested the following five different situations which might give rise to breaks in regular, rigid routine behavior or path dependency: 
- encountering a novel state of affairs

- experiencing a failure

- reaching a milestone in the life of work

- receiving an intervention that calls members' attention to their own group norms

- having to cope with change in the structure of the group

Some researchers, for example Cohen et al. (1996), emphasize external influences. They propose that routines are fixed until an external event causes them to change. On the other hand, Feldman (2000) has shown that routines can change (be broken) endogenously when the routine act results in undesirable, unintended outcomes. Today, undesirable, unintended outcomes from the psychiatric field have become a burden for many societies (Allen Frances, 2009). It is time to break away from routines and path dependency, giving room for the following improvements in psychiatry (Sjödahl, 2012):

- better diagnostics, also including causal analyses

- better treatment alternatives, meaning cooperation between different subject specialists

- better follow-up studies of single cases, planning rehabilitation opportunities

- better task feedback to diagnosing clinicians to prevent iatrogenic damage better working conditions for clinical personal making practice based learning posssible

References:

Adevi, A. A. (2012). Supportive nature - and stress. Wellbeing in connection to our inner and outer landscape. Doctoral thesis: Swedish University of Agricultural Sciences, (11).

Arthur, B. W. (1994). Increasing returns and path dependence in the economy. Ann Arbor MI: University of Michigan Press.

Bennett, A., \& Elman, C. (2006). Complex causal relations and case study methods: The example of path dependence. Political Analysis, 14, 250-267.

Brunswik, E. (1956). Perception and the representative design of psychological experiments. Berkeley and Los Angeles, California: University of California Press.

Cohen, M. D. Burkhart, R., Dosi, G., et al. (1996). Routines and other recurring action patterns of organizations: Contemporary research issues. Industrial \& Corporate Change, 5(3), 653-699.

Cyert, R. M., \& March, J. G. (1963/1992). A behavioral theory of the firm (second ed.). Englewood Cliffs, NJ: Prentice-Hall.

Feldman, M. S. (2000). Organizational routines as a source of continuous change. Organization Science, 11(6), 611-629.

Fiedler, K., \& Juslin, P. (2006). Information sampling and adaptive cognition. Cambridge, New York, Melbourne: Cambridge University Press.

Flanagan, J. (1954). The critical incident technique. Psychological Bulletin, 51(4), 327-358.

Frances, A. (2009). A warning sign on the road to DSM-V. Beware of its unintended consequences. Psychiatric Times 26(8). Available at: http://www.psychiatrictimes.com/articles/warning-sign-roaddsm-v-beware-its-unintended-consequences

Frances, A. (2010). Opening Pandora's box. The worst suggestions for DSM5. Psychiatric Times. February 11.

Gerrick, C. J. G., \& Hackman, J. R. (1990). Habitual routines in task-performing groups. Organizational Behavior and Human Decision Processes, 47(1), 65-98.

Gigerenzer, G. (2002). Reckoning with risk. Learning to live with uncertainty. London. Penguin Books.

Groenier, M., Pieters, J. M., Hulshof, C. D., \& al. (2008). Psychologists' judgements of diagnostic activities: Deviations from a theoretical model. Clinical Psychology and Psychotherapy, 15, 256265. 
Kahneman, D., \& Lowallo, D. (1993). Timid choices and bold forecasts: A cognitive perspective on risk taking. Management Science, 39(1), 17-31.

Kirk, S. A., \& Kutchins, H. (1992). The selling of DSM. The rhetoric of science in psychiatry. New York: Aldine de Gruyter.

Leonard-Barron, D. (1992). Core capabilities and core rigidities: A paradox in managing new product development. Strategic Management Journal, 13, 111-125.

Levinthal, D., \& Myatt, J. (1994). Co-evolution of capabilities and industry. The evolution of mutual fund processing. Strategic Management Journal, 15, 45-62.

March, J. G. (1994). A primer on decision making: How decisions happen. New York: Free Press.

Mintzberg, H., Raisinghani, D., \& Théorét, A. (1976). The structure of "unstructured" decision processes. Administrative Science Quarterly, 21(2), 246-275.

Nelson, R. R., \& Winter, S. G. (1982). An evolutionary theory of economic change. Cambridge: The Belknap Press of Harvard University Press.

Nussbaum, M. C. (2011). Creating capabilities. The human development approach. Cambridge, Massachusetts, London, England: The Belknap Press of Harvard University Press.

Örstadius, K. (2012). Anmälningar mot läkare lagts på hög. Dagens Nyheter 10 February. (in Swedish).

Simon, H. A. (1947/1997). Administrative behavior: A study of decision-making processes in administrative organization $\left(4^{\mathrm{ed}}\right)$. New York: The Free Press.

Sjödahl, L. (2011). The vagueness of creativity. Reflections on some Brunswik concepts. The Brunswik Society Newsletter, 26, 4.

Sjödahl, L. (2012). Lose your forest and you lose everything. The Brunswik Society Newsletter, $27,31$.

Sjödahl, L. (2012). Psychiatric diagnoses - are they to be trusted? First listen to the patient! Visby, Sweden, Nomen Publishing.

Swedish National Board of Health and Welfare (1997). Report 8, 61-62. (In Swedish). Von Wright, G. H. (1971). Explanation and understanding. London: Routledge \& Kegan Paul. 


\title{
The Brunswik Society News/etter, 2013
}

\section{Using Lens Model Analysis to Understand Impression Formation in Online Social Networks}

\author{
Juliane M. Stopfer \\ Georg August University Göttingen, \\ GER \\ Boris Egloff \\ Johannes Gutenberg University Mainz, \\ GER \\ Steffen Nestler \& Mitja D. Back \\ Westfälische Wilhelms University Münster, \\ GER
}

Contacts: jstopfer@uni-goettingen.de / steffen.nestler@wwu.de / mitja.back@wwu.de

How do people form first impressions of others' personality? Why do people spontaneously like one person, but not the other? Understanding the processes of impression formation is essential as first impressions influence people's daily decision making and may have long-lasting consequences. In recent years, social and personality psychologists have shed light on the accuracies and inaccuracies in judging certain personality traits on the basis of various sources (e.g., offices and bedrooms, email addresses, music preferences; see Funder, 2012, and Gosling, 2008, for overviews). In many of these studies, behavioral cues were examined as mediators for explaining the accuracy of judgments (cf. lens model; Brunswik, 1956; see also Nestler \& Back, 2012, 2013): For example, in a study by Borkenau and colleagues (Borkenau, Brecke, Möttig, \& Paelecke, 2009), perceivers accurately judged extraversion from facial images. This finding is explained by (a) targets' extraversion expressing itself in facial cues (i.e., cue validity) and (b) lay perceivers basing their extraversion impressions on perceivable facial cues (i.e., cue utilization). Perceivers used those cues for impression formation that were actually related to the targets' personality (e.g., cheerful facial expression), leading to accurate judgments.

In our own research (Back et al., 2010; Stopfer, Egloff, Nestler, \& Back, in press; Stopfer, Egloff, Nestler, \& Back, 2013), we investigated first impressions in a context that plays an essential role in people's everyday social lives: online social networks (OSNs; for example Facebook). In OSNs, users present themselves in terms of a personal OSN profile (including a profile picture, a group list, etc.) and can interact with others in numerous ways like posting a message on another user's notice board (see for example, Stopfer \& Gosling, 2013, for a more detailed definition of OSNs). As OSNs allow for fine-grained analyses of all presented behaviors (e.g., befriending others, posting on others' notice boards), the concrete processes involved in personality expression and interpersonal perception can be examined in detail. Using OSN profiles, we applied and extended the above mentioned lens model approach to understanding impression formation in various ways:

First, on the basis of Brunswik's concept of vicarious functioning (Brunswik, 1956; see Cooksey, 1996, for an overview), cues were thought to be (at least partly) 


\section{The Brunswik Society News/etter, 2013}

substitutable for one another because of their intercorrelations (i.e., cues are redundant regarding what they indicate). Thus, we assumed that accuracy occurs even if restricted (sets of) cues were utilized for impression formation; for example, when judgments were made on the basis of reduced information only (thin slices of OSN profiles). Our findings confirmed this assumption: Openness to experience was accurately judged by different groups of perceivers who viewed different thin slices of OSN profiles (profile picture, interest field, group list, notice board).

Second, we hypothesized that cues could be represented as both circumscribed behaviors (e.g., a creative OSN profile picture as a cue to infer openness) and thinslice impressions (e.g., the openness judgment based on a user's profile picture as a cue for an overall impression of openness based on a user's full OSN profile). This idea was borrowed from the hierarchical judgment design where judgments made within one hierarchical layer lay the foundation for the cue values for judgments in the next layer (Cooksey, 1996; Hammond, Stewart, Brehmer, \& Steinmann, 1975). Again, our assumption was proved: The accuracy of judging openness on the basis of OSN profiles was mediated by both accurate thin-slice impressions (e.g., based on the profile picture) and valid behavioral cues (e.g., creative picture).

Third, we also applied the lens model approach towards understanding impression management and meta-accuracy as phenomena that are based on cueexpression and cue-perception processes: Our results showed that targets expressed their desired impressions (how they wished to be viewed by others on the basis of their OSN profiles) in terms of behavioral OSN cues (e.g., a self-promotional group list) and perceivers, in part, utilized these cues for impression formation, leading to distorted personality impressions. For meta-accuracy (knowing how one is viewed by others), we were able to show that both targets and perceivers utilized behavioral cues for impression formation: For extraversion, targets utilized cues to infer how others might judge them on the basis of their OSN profiles (e.g., the extent of detail of the OSN interests field), and perceivers utilized the very same cues to judge the targets' personality, leading to meta-accuracy.

Finally, we investigated how personality differentially affected peer-perceived popularity (status) and sociometric popularity (liking) and why. Our results showed that targets scoring high on agency were ascribed a high status, whereas targets scoring high on creativity or communion were liked. Brunswikian lens model analyses revealed mediating behavioral cues: For example, agentic targets tended to have many entries from other sex peers on their notice boards; perceivers, on the other hand, utilized the number of notice boards entries from other sex peers to infer a target's status, explaining the agency-status link. In sum, the Brunswikian lens model can serve as a valuable tool for fostering our understanding of the processes of expressing personality and judging personality and popularity. We wish to encourage researchers to continue applying and extending the lens model approach to understand meaningful social phenomena.

References:

Back, M. D., Stopfer, J. M., Vazire, S., Gaddis, S., Schmukle, S. C., Egloff, B., \& Gosling, S. D. (2010). Facebook profiles reflect actual personality, not self-idealization. Psychological Science, 21, 372374.

Borkenau, P., Brecke, S., Möttig, C., \& Paelecke, M. (2009). Extraversion is accurately perceived after a 50-ms exposure to a face. Journal of Research in Personality, 43, 703-706. 


\title{
The Brunswik Sogiety

Brunswik, E. (1956). Perception and the representative design of psychological experiments $\left(2^{\text {nd }}\right.$ ed.). Berkeley, CA: University of California Press.

Cooksey, R. W. (1996). Judgment analysis: Theory, methods, and applications. San Diego, CA: Academic Press.

Funder, D. C. (2012). Accurate personality judgments. Current Directions in Psychological Science, 21, 177-182.

Gosling, S. D. (2008). Snoop: What your stuff says about you. New York, NY: Basic Books.

Nestler, S., \& Back, M. D. (2012). Applications and extensions of lens model analyses in personality psychology. The Brunswik Society Newsletter, 27, 32-34.

Nestler, S., \& Back, M. D. (2013). Applications and extensions of the lens model to understand interpersonal judgments at zero acquaintance. Current Directions in Psychological Science, 22, 374-379.

Hammond, K. R., Stewart, T. R., Brehmer, B., \& Steinmann, D. O. (1975). Social judgment theory. In M. Kaplan \& S. Schwartz (Eds.), Human judgment and decision processes (pp. 271-312). New York, NY: Academic Press.

Stopfer, J. M., Egloff, B., Nestler, S., \& Back, M. D. (in press). Personality expression and impression formation in online social networks: An integrative approach to understanding the processes of accuracy, impression management, and meta-accuracy. European Journal of Personality.

Stopfer, J. M., Egloff, B., Nestler, S., \& Back, M. D. (2013). Being popular in online social networks: How agentic, communal, and creativity traits relate to judgments of status and liking. Journal of Research in Personality, 47, 592-598.

Stopfer, J. M., \& Gosling, S. D. (2013). Online social networks in the work context. In D. Derks \& A. Bakker (Eds.), The psychology of digital media at work. Hove, UK: Psychology Press.

\section{A Lens Model Approach to Explain Fluency Effects in Judgments and Decisions}

\author{
Christian Unkelbach \\ University of Cologne, \\ GER \\ Rainer Greifeneder \\ University of Basel, \\ $\mathrm{CH}$
}

Contacts: christian.unkelbach@uni-koeln.de / rainer.greifeneder@unibas.ch

Processing fluency is the experienced ease of ongoing cognitive processes; people experience this ease from perception, from retrieval, or from generation of information. In other words, mental processes vary on a dimension from easy to difficult, from fluent to disfluent. For example, it is easier to read this Word compared to this Word, and it is easier to calculate $3 \times 3$ than to calculate $17 \times 24$.

Importantly, this fluency experience influences many judgments and decisions: fluently perceived names are judged as famous, fluently read statements are evaluated as true, and fluently retrieved events from memory are estimated to be likely and frequent. The latter example is probably the most famous example of fluency effects in judgments, provided by Tversky and Kahneman (1973). In their seminal study, participants judged the frequency of words starting with " $r$ " and words that have " $r$ " as the third letter. Although objectively, the latter words are more frequent, participants judged the former words as more frequent, simply because these words come easier to mind. Forty years later it seems almost self-evident that frequency is judged via retrieval fluency (or availability, to use Tversky and Kahneman's term). Yet, 


\section{The Brunswilk Society

fluency experiences influence many judgments about other properties of the empirical world, such as fame (Jacoby, Kelley, Brown, \& Jasechko, 1989), ability (Greifeneder et al., 2010), the economic value of stocks (Alter \& Oppenheimer, 2006), the gross value of companies (Hertwig, Herzog, Schooler, \& Reimer, 2008), or the truth of statements (Unkelbach, 2007).

The open empirical question is how one, at least theoretically, unitary experience influences judgments about so many different aspects of the empirical world. To answer this question, we used Brunswik's lens model to formulate a general model of fluency effects (Unkelbach \& Greifeneder, 2013). What all this properties have in common is that they are distal concepts; there is no sensory input that allows to judge fame, economic value, or truth. Thus, people must rely on proximal cues to judge these distal properties. A prominent example is truth: to judge the truth of a statement, people can rely on coherence, preciseness, immediacy, source credibility, and vividness. For our model, we assumed that individuals use fluency in a similar way as a proximal cue to form judgments about distal criteria - such as liking, frequency, or truth. In other words, fluency is a general metacognitive cue that is common to all the examples provided above.

As the lens model has two sides, the ecological correlation and the cue utilization, it is possible to test the model that fluency functions as a general metacognitive cue. A number of experiments manipulated the correlation of the cue with the distal criterion. In other words, they created learning environments in which high fluency correlated with the opposite of the standard effect (e.g., unfamiliarity, falseness). Indeed, when the correlation of fluency with the criterion changed and people were able to learn this via feedback, the influence on judgments reversed as well (Unkelbach, 2006, 2007; Unkelbach \& Stahl, 2009).

These experiments represent an idealized world in which a cue's ecological correlation (i.e., its validity) indeed determines the cue's utilization (i.e., its weight in a judgment). However, in many cases, ecological correlation and cue utilization do not match (Karelaia \& Hogarth, 2008); for the fluency case, these are the instances when the influence of fluency is rightfully termed a bias or an unwanted influence; for example, when statements are believed because one has heard them before (e.g., Begg, Anas, \& Farinacci, 1992), or when stocks are rated higher simply because their names are easy to pronounce (Alter \& Oppenheimer, 2006). Nevertheless, we believe it highly fruitful to conceptualize processing fluency as a metacognitive cue within the lens model framework. It allows other cues to be integrated into judgments in a linear fashion (in contrast to a fluency heuristic view; Hertwig et al., 2008) and it allows for the cue utilization to change when the ecological correlation changes (in contrast to naïve theories; Schwarz, 2004). In addition, it emphasizes the adaptive nature of human cognition instead of the possible pitfalls and biases in human reasoning. Foremost, the lens model approach explains how fluency influences so many distinct properties environment, such as liking, fame, truth, ability, justice, or economic value. Simply because people have no directly available informative about these properties, they must rely on proximal cues; and the experienced ease of ongoing cognitive processes, that is, processing fluency, might be one of the most prominent and readily available cues. 
References:

Alter, A. L., \& Oppenheimer, D. M. (2006). Predicting short-term stock fluctuations using processing fluency. Proceedings of the National Academy of Sciences, USA, 103, 9369-9372.

Begg, I. M., Anas, A., \& Farinacci, S. (1992). Dissociation of processes in belief: Source recollection, statement familiarity, and the illusion of truth. Journal of Experimental Psychology: General, 121, 446-458.

Greifeneder, R., Alt, A., Bottenberg, K., Seele, T., Zelt, S., \& Wagener, D. (2010). On writing legibly: Processing fluency systematically biases evaluations of handwritten material. Social Psychological and Personality Science, 1, 230-237.

Hertwig, R., Herzog, S. M., Schooler, L. J., \& Reimer, T. (2008). Fluency heuristic: A model of how the mind exploits a by-product of information retrieval. Journal of Experimental Psychology: Learning, Memory, and Cognition, 34, 1191-1206.

Jacoby, L. L., Kelley, C., Brown, J., \& Jasechko, J. (1989). Becoming famous overnight: Limits on the ability to avoid unconscious influences of the past. Journal of Personality and Social Psychology, 56, 326-338.

Karelaia, N., \& Hogarth, R. (2008). Determinants of linear judgment: A meta-analysis of lens model studies. Psychological Bulletin, 134, 404-426.

Schwarz, N. (2004). Meta-cognitive experiences in consumer judgment and decision making. Journal of Consumer Research, 14, 332-348.

Tversky, A., \& Kahneman, D. (1973). Availability: A heuristic for judging frequency and probability. Cognitive Psychology, 5, 207-232.

Unkelbach, C. (2006). The learned interpretation of cognitive fluency. Psychological Science, 17, 339345.

Unkelbach, C. (2007). Reversing the truth effect: Learning the interpretation of processing fluency in judgments of truth. Journal of Experimental Psychology: Learning, Memory and Cognition, 33, 219230.

Unkelbach, C., \& Greifeneder, R. (2013). A general model of fluency effects in judgment and decision making. In C. Unkelbach \& R. Greifeneder (Eds.), The Experience of Thinking. London: Psychology Press.

Unkelbach, C., \& Stahl, C. (2009). A multinomial modeling approach to dissociate different components of the truth effect. Consciousness and Cognition, 18, 22-38. 


\section{The Brunswik Society Newsletter, 2013}

To get information about future Newsletters you are welcome to join (free of charge) the email list of the Brunswik Society at:

htțp://www.brunswik.org/ 